\title{
Unitarization and Inversion Formulae for the Radon Transform between Dual Pairs
}

\author{
Giovanni S. Alberti* \\ Francesca Bartolucci* \\ Filippo De Mari* \\ Ernesto De Vito*
}

October 31, 2018

\begin{abstract}
We consider the Radon transform associated to dual pairs $(X, \Xi)$ in the sense of Helgason, with $X=G / K$ and $\Xi=G / H$, where $G=\mathbb{R}^{d} \rtimes K, K$ is a closed subgroup of $\operatorname{GL}(d, \mathbb{R})$ and $H$ is a closed subgroup of $G$. Under some technical assumptions, we prove that if the quasi regular representations of $G$ acting on $L^{2}(X)$ and $L^{2}(\Xi)$ are irreducible, then the Radon transform admits a unitarization intertwining the two representations. If, in addition, the representations are square integrable, we provide an inversion formula for the Radon transform based on the voice transform associated to these representations.

Key words. Homogeneous spaces, Radon transform, spherical means Radon transform, dual pairs, square-integrable representations, inversion formula, wavelets, shearlets.
\end{abstract}

Mathematics Subject Classification. 44A12, 42C40, 22D10.

\section{Introduction}

In a remarkable series of papers (see, e.g., [19, 18]), for the most part subsumed in the monographs [21, 22, 20, 23], Helgason has developed a broad theory of Radon transforms in a differential geometric setup. In this paper we show that the above framework is particularly appropriate in order to treat in a unified way some results concerning unitarizability features and inversion formulae of various types of Radon transforms [5, 24] and permits to handle a significant number of other interesting examples.

One of the central notions in Helgason's theory is that of dual pair $(G / K, G / H)$ of homogeneous spaces of the same Lie group $G$, where $K$ and $H$ are closed subgroups of $G$. The transitive $G$-space $X=G / K$ is meant to describe the ambient in which the functions to be analyzed live, prototypically a space of constant curvature like the Euclidean plane, or the sphere $S^{2}$ or the hyperbolic plane $\mathbb{H}^{2}$. A large and important part of Helgason's work is devoted to the case when $X$ is actually a symmetric space, whence the notation $G / K$ that we retain. The second transitive $G$-space $\Xi=G / H$ is meant to parametrize the set of submanifolds of $X$ over which one wants to integrate

*Department of Mathematics, University of Genoa, Via Dodecaneso 35, 16146 Genova, Italy (alberti@dima.unige.it, bartolucci@dima.unige.it, demari@dima.unige.it, devito@dima.unige.it). 
functions, for instance hyperplanes in Euclidean space, great circles in $S^{2}$, geodesics or horocycles in $\mathbb{H}^{2}$. With this basic understanding in mind, the notion of incidence between $x \in X$ and $\xi \in \Xi$ translates the intuition that $x=g_{1} K$ is a point of $\xi=g_{2} H$ and amounts to the fact that $g_{1} K \cap g_{2} H \neq \emptyset$. In this way any element $\xi \in \Xi$ may be realized as a submanifold $\hat{\xi} \subset X$ simply by taking all the points $x \in X$ that are incident to $\xi$; conversely, one builds the "sheaf of manifolds" $\check{x}$ through the point $x \in X$ by taking all the points $\xi \in \Xi$ that are incident to $x$. If the maps $\xi \mapsto \hat{\xi}$ and $x \mapsto \check{x}$ are injective, then $(X, \Xi)$ is a dual pair. Under this assumption, the Radon transform $\mathcal{R}$ takes functions on $X$ into functions on $\Xi$ and is abstractly defined by

$$
\mathcal{R} f(\xi)=\int_{\hat{\xi}} f(x) \mathrm{d} m_{\xi}(x),
$$

provided that, for all $\xi \in \Xi, m_{\xi}$ is a suitable measure on the manifold $\hat{\xi}$ and the right hand side is meaningful, possibly in some weak sense. The first requirement is achieved by observing that, denoted by $\xi_{0}=e H$ the origin of $\Xi$, it is easy to check that $\widehat{\xi_{0}} \subset X$ is actually a transitive $H$-space, hence $\widehat{\xi_{0}}$ carries a measure $m_{0}$ which is quasi-invariant with respect to the $H$-action [30]. Via the $G$-action, for all $\xi \in \Xi$ the measures $\left\{m_{\xi}\right\}$ on $\{\hat{\xi}\}$ are defined as the push-forward of $m_{0}$.

As for the right space of functions $f: X \rightarrow \mathbb{C}$ for which the Radon transform makes sense, a natural choice is the $L^{2}$ setting. Indeed, both $X$ and $\Xi$ are transitive spaces, so that there exist quasi-invariant measures $\mathrm{d} x$ and $\mathrm{d} \xi$. In this context, a central issue is to prove that the Radon transform, up to a composition with a suitable pseudodifferential operator, can be extended to a unitary map $\mathcal{Q}$ from $L^{2}(X, \mathrm{~d} x)$ to $L^{2}(\Xi, \mathrm{d} \xi)$ intertwining the quasi-regular representations $\pi$ and $\hat{\pi}$ of $G$, which naturally act on $L^{2}(X, \mathrm{~d} x)$ and $L^{2}(\Xi, \mathrm{d} \xi)$, respectively. We stress that in Helgason's approach, it is assumed that $m_{0}, \mathrm{~d} x$ and $\mathrm{d} \xi$ are all invariant measures. The reader is again referred to Helgason's books for a thorough treatment, as well as for the broad problem of the operator properties of $\mathcal{R}$.

In this paper we address the special case in which the group $G$ is a semidirect product of the form $\mathbb{R}^{d} \rtimes K$, where $K$ is a closed subgroup of $\operatorname{GL}(d, \mathbb{R})$, and the representations $\pi$ and $\hat{\pi}$ are both irreducible. Under some technical assumptions that we describe below, we prove a unitarization result, see Theorem 5 . The proof is based on the generalization of Shur's lemma provided by Duflo and Moore [12. One of the novelties of our treatment consists in making weaker assumptions on $m_{0}, \mathrm{~d} x$ and $\mathrm{d} \xi$, namely their relative invariance instead of invariance. This allows for considering a wider variety of cases, such as wavelets and shearlets. A well-known predecessor of Theorem [5] is Theorem 4.1 in [20], an alternative proof of which, tailored to our particular viewpoint, is to be found in [5].

If, in addition, we require that $\pi$ is square integrable (so that $\hat{\pi}$ is square integrable, too) we derive a general inversion formula for $\mathcal{R}$ of the form

$$
f=\int_{G} \chi(g)\langle\mathcal{R} f, \hat{\pi}(g) \Psi\rangle \pi(g) \psi \mathrm{d} \mu(g),
$$

where $\chi$ is a character of $G$ and $\psi \in L^{2}(X, \mathrm{~d} x)$ and $\Psi \in L^{2}(\Xi, \mathrm{d} \xi)$ are suitable mother wavelets and the Haar integral is weakly convergent, see Theorem 6 . We stress that the coefficients $\langle\mathcal{R} f, \hat{\pi}(g) \Psi\rangle$ depend on $f$ only through its Radon transform $\mathcal{R} f$, so that the above equation allows to reconstruct an unknown signal from its Radon transform by computing the family of coefficients $\{\langle\mathcal{R} f, \hat{\pi}(g) \Psi\rangle\}_{g \in G}$. As it is clear from (1), $\hat{\pi}$ 
is used as an "analysis" transform applied to $\mathcal{R} f$ and $\pi$ as a "synthesis" transform to reconstruct $f$. This kind of reconstruction formulae is already known for the classical Radon trasform where $G$ is the affine group of $\mathbb{R}^{d}$ associated with the multi-dimensional wavelets, [24, 31, 28, 7, 27, and for the affine Radon transform where $G$ is the shearlet group [5].

We illustrate the construction and the result with the examples where $G$ is either the similitude group of the plane (with two different choices of $\Xi$ ) or the standard shearlet group [26], but other cases could also be covered, such as the generalized shearlet dilation groups [16, 2]. We believe that our contribution may be further substantiated with several other examples and deepened in several directions. In particular, it would be interesting to consider more general groups $G$ and to relax the assumption of irreducibility of the representations. This would allow us to include many other examples, such as the class of groups studied in [1, 3].

For clarity, we list the main assumptions that are made along the way. We consider:

- a Lie group $G=\mathbb{R}^{d} \rtimes K$, where $K$ is a closed subgroup of $\operatorname{GL}(d, \mathbb{R})$;

- the space $X=\mathbb{R}^{d}$, regarded as a transitive $G$-space with respect to the canonical action of $G$ (denoted by $(b, k)[x]=b+k x$ for $(b, k) \in G$ and $x \in X$ ), equipped with the Lebesgue measure $\mathrm{d} x$;

- a smooth transitive $G$-space $\Xi$ (we denote the action of $G$ on $\Xi$ by $g . \xi$ ) with an origin $\xi_{0} \in \Xi$ and $H$ the isotropy at $\xi_{0}$;

and we assume that the following conditions hold true:

(A1) the space $\Xi$ carries a relatively $G$-invariant measure $\mathrm{d} \xi$;

(A2) denoting the origin of $X$ by $x_{0}=0$, the $H$-transitive space

$$
\hat{\xi}_{0}=H\left[x_{0}\right] \subset X
$$

carries a relatively $H$-invariant measure $m_{0}$ with character $\gamma$;

(A3) there exists a Borel section $\sigma: \Xi \rightarrow G$ such that

$$
(g, \xi) \mapsto \gamma\left(\sigma(\xi)^{-1} g \sigma\left(g^{-1} . \xi\right)\right)
$$

extends to a positive character of $G$ independent of $\xi$;

(A4) the pair $(X, \Xi)$ is a dual pair in the sense of Helgason under the canonical isomorphism $X=G / K$ and $\Xi=G / H$;

(A5) the quasi-regular representation $\pi$ of $G$ acting on $L^{2}(X, \mathrm{~d} x)$ is irreducible and square-integrable;

(A6) the quasi-regular representation $\hat{\pi}$ of $G$ acting on $L^{2}(\Xi, \mathrm{d} \xi)$ is irreducible;

(A7) there exists a non-trivial $\pi$-invariant subspace $\mathcal{A} \subseteq L^{2}(X, \mathrm{~d} x)$ such that

$$
\begin{aligned}
& f(\sigma(\xi)[\cdot]) \in L^{1}\left(\hat{\xi}_{0}, m_{0}\right) \quad \text { for almost all } \xi \in \Xi, \\
& \mathcal{R} f:=\int_{\hat{\xi}_{0}} f(\sigma(\cdot)[x]) \mathrm{d} m_{0}(x) \in L^{2}(\Xi, \mathrm{d} \xi),
\end{aligned}
$$

for all $f \in \mathcal{A}$, and the map $f \mapsto \mathcal{R} f$ is a closable operator from $\mathcal{A}$ to $L^{2}(\Xi, \mathrm{d} \xi)$.

We note that the condition that $(X, \Xi)$ is a dual pair (assumption (A4) will not play any explicit role, as it is implied by (A5) and (A6) (see Remark 1 below). The assumption that $\pi$ is square-integrable is needed only in Section 4 , whereas the condition that $\hat{\pi}$ is irreducible is not needed until Theorem 5 . 


\section{Preliminaries}

\section{$2.1 \quad$ Notation}

We briefly introduce the notation. We set $\mathbb{R}^{\times}=\mathbb{R} \backslash\{0\}$ and $\mathbb{R}^{+}=(0,+\infty)$. The Euclidean norm of a vector $v \in \mathbb{R}^{d}$ is denoted by $|v|$ and its scalar product with $w \in \mathbb{R}^{d}$ by $v \cdot w$. For any $p \in[1,+\infty]$ we denote by $L^{p}\left(\mathbb{R}^{d}\right)$ the Banach space of functions $f: \mathbb{R}^{d} \rightarrow \mathbb{C}$ that are $p$-integrable with respect to the Lebesgue measure $\mathrm{d} x$ and, if $p=2$, the corresponding scalar product and norm are $\langle\cdot, \cdot\rangle$ and $\|\cdot\|$, respectively. If $E$ is a Borel subset of $\mathbb{R}^{d},|E|$ also denotes its Lebesgue measure. The Fourier trasform is denoted by $\mathcal{F}$ both on $L^{2}\left(\mathbb{R}^{d}\right)$ and on $L^{1}\left(\mathbb{R}^{d}\right)$, where it is defined by

$$
\mathcal{F} f(\xi)=\int_{\mathbb{R}^{d}} f(x) \mathrm{e}^{-2 \pi i \xi \cdot x} \mathrm{~d} x, \quad f \in L^{1}\left(\mathbb{R}^{d}\right) .
$$

If $G$ is a Lie group, we denote by $L^{2}(G)$ the Hilbert space of square-integrable functions with respect to a left Haar measure on $G$. If $X$ is a smooth transitive $G$-space with origin $x_{0}$, denoted by $g[x]$ the action of $G$ on $X$, a Borel measure $\mu$ of $X$ is relatively invariant if there exists a positive character $\alpha$ of $G$ such that for any measurable set $E \subset X$ and $g \in G$ it holds $\mu(g[E])=\alpha(g) \mu(E)$. Furthermore, a Borel section is a measurable map $s: X \rightarrow G$ satisfying $s(x)\left[x_{0}\right]=x$ and $s\left(x_{0}\right)=e$, with $e$ the neutral element of $G$; a Borel section always exists since $G$ is second countable [30, Theorem 5.11]. We denote the (real) general linear group of size $d \times d$ by GL( $d, \mathbb{R})$.

\subsection{Dual homogeneous spaces of semidirect products}

In this section we recall the basic construct due to Helgason [20] adapted to the context in which we are interested, namely when the group $G$ is the semidirect product of the Euclidean space $\mathbb{R}^{d}$ with a closed subgroup $K$ of $G \mathrm{~L}(d, \mathbb{R})$. This structure is enjoyed by several groups of interest in applications, such as the similitude group studied by Murenzi [4, and the generalized shearlet dilation groups introduced by Führ in [14, 16] for the purpose of generalizing the standard shearlet group introduced in [26, 10]. Whenever possible, we keep the notation as in 20 .

We recall that $G=\mathbb{R}^{d} \rtimes K$ is the manifold $\mathbb{R}^{d} \times K$ endowed with the group operation

$$
\left(b_{1}, k_{1}\right)\left(b_{2}, k_{2}\right)=\left(b_{1}+k_{1} b_{2}, k_{1} k_{2}\right), \quad b_{1}, b_{2} \in \mathbb{R}^{d}, k_{1}, k_{2} \in K,
$$

where $k b$ is the natural linear action of the matrix $k$ on the column vector $b$, so that $G$ is a Lie group. The inverse of an element in $G$ is given by $(b, k)^{-1}=\left(-k^{-1} b, k^{-1}\right)$. A left Haar measure of $G$ is

$$
\mathrm{d} \mu(b, k)=|\operatorname{det} k|^{-1} \mathrm{~d} b \mathrm{~d} k,
$$

where $\mathrm{d} b$ is the Lebesgue measure of $\mathbb{R}^{d}$ and $\mathrm{d} k$ is a left Haar measure on $K$.

The first transitive space we consider is $X=\mathbb{R}^{d}$, regarded as smooth $G$-space with respect to the canonical action

$$
(b, k)[x]=b+k x, \quad(b, k) \in G, x \in X .
$$

The action is clearly transitive, the isotropy at the origin $x_{0}=0$ is the subgroup $\{(0, k): k \in K\}$ which we identify with $K$, so that $X \simeq G / K$. Furthermore, the map

$$
s: X \rightarrow G, \quad s(x)=\left(x, \mathrm{I}_{d}\right),
$$


is a Borel section and the Lebesgue measure $\mathrm{d} x$ on $X$ is a relatively $G$-invariant measure, since for any measurable set $E \subseteq \mathbb{R}^{d}$ we have

$$
|(b, k)[E]|=|b+k E|=|k E|=|\operatorname{det} k||E| .
$$

We consider a second smooth transitive $G$-space $\Xi$, whose action is denoted by

$$
G \times \Xi \ni(g, \xi) \mapsto g . \xi \in \Xi .
$$

By Assumption (A1), $\Xi$ admits a relatively invariant measure $\mathrm{d} \xi$, which may be expressed by the equality

$$
\int_{\Xi} f\left(g^{-1} \cdot \xi\right) \mathrm{d} \xi=\beta(g) \int_{\Xi} f(\xi) \mathrm{d} \xi, \quad f \in L^{1}(\Xi, \mathrm{d} \xi), g \in G,
$$

where $\beta: G \rightarrow(0,+\infty)$ is a positive character of $G$.

We fix an origin $\xi_{0} \in \Xi$, we denote the isotropy at $\xi_{0}$ by $H$ and we put

$$
\check{x}_{0}=K . \xi_{0} \subset \Xi, \quad \hat{\xi}_{0}=H\left[x_{0}\right] \subset X .
$$

By definition, $\check{x}_{0}$ and $\hat{\xi}_{0}$ are $K$ and $H$ transitive spaces, respectively. In order to define the Radon transform we will make use of Assumption (A2), namely that $\hat{\xi}_{0}$ carries a relatively $H$-invariant Radon measure $\mathrm{d} m_{0}$, that is

$$
\int_{\hat{\xi}_{0}} f\left(h^{-1}[x]\right) \mathrm{d} m_{0}(x)=\gamma(h) \int_{\hat{\xi}_{0}} f(x) \mathrm{d} m_{0}(x), \quad f \in L^{1}\left(\hat{\xi_{0}}, \mathrm{~d} m_{0}\right), h \in H,
$$

where $\gamma: H \rightarrow(0,+\infty)$ is a positive character of $H$. This is a weaker assumption than in Helgason's approach, in which $\hat{\xi}_{0}$ is assumed to admit a bona fide invariant measure for the $H$-action.

We fix a Borel section $\sigma: \Xi \rightarrow G$ such that (A3) holds true. With an equivalent approach to that of Helgason's, we define the sets

$$
\hat{\xi}=\sigma(\xi)\left[\hat{\xi}_{0}\right] \subset X, \quad \check{x}=s(x) . \check{x}_{0} \subset \Xi,
$$

which are closed subsets by [20, Lemma 1.1].

Assumption (A4) states that $(X, \Xi)$ is a dual pair, which means that the maps $x \mapsto \check{x}$ and $\xi \mapsto \hat{\xi}$ are both injective. This assumption is called transversality, see [20, Lemma 1.3] about an equivalent characterization. Apart from the cases considered below, the reader may consult [20] for numerous examples of dual pairs $(X, \Xi)$.

Remark 1. As mentioned in the introduction, transversality is in fact implied by assumptions (A5) and (A6) (we leave this investigation for more general groups and spaces to future work) and will never be used in the arguments below.

The following example shows that the (classical) Radon transform can be obtained in this framework. Two other examples are illustrated in Section 5 .

Example 1. The (connected component of the identity of the) similitude group $\operatorname{SIM}(2)$ of the plane is $\mathbb{R}^{2} \rtimes K$, with $K=\left\{R_{\phi} A_{a} \in \mathrm{GL}(2, \mathbb{R}): \phi \in[0,2 \pi), a \in \mathbb{R}^{+}\right\}$ where

$$
R_{\phi}=\left[\begin{array}{cc}
\cos \phi & -\sin \phi \\
\sin \phi & \cos \phi
\end{array}\right], \quad A_{a}=\left[\begin{array}{cc}
a & 0 \\
0 & a
\end{array}\right] .
$$


By the identification $K \simeq[0,2 \pi) \times \mathbb{R}^{+}$, we write $(b, \phi, a)$ for the elements in $\operatorname{SIM}(2)$. With this identification the group law becomes

$$
(b, \phi, a)\left(b^{\prime}, \phi^{\prime}, a^{\prime}\right)=\left(b+R_{\phi} A_{a} b^{\prime}, \phi+\phi^{\prime} \bmod 2 \pi, a a^{\prime}\right),
$$

and the inverse of $(b, \phi, a)$ is given by

$$
(b, \phi, a)^{-1}=\left(-A_{a}^{-1} R_{\phi}^{-1} b,-\phi \bmod 2 \pi, a^{-1}\right) .
$$

By (2), a left Haar measure of $\operatorname{SIM}(2)$ is

$$
\mathrm{d} \mu(b, \phi, a)=a^{-3} \mathrm{~d} b \mathrm{~d} \phi \mathrm{d} a,
$$

where $\mathrm{d} b, \mathrm{~d} \phi$ and $\mathrm{d} a$ are the Lebesgue measures on $\mathbb{R}^{2},[0,2 \pi)$ and $\mathbb{R}_{+}$, respectively.

It remains to choose the space $\Xi$ and the corresponding subgroup $H$ of $S I M(2)$. The group $\operatorname{SIM}(2)$ acts transitively on $\Xi=[0, \pi) \times \mathbb{R}$ by

$$
(b, \phi, a) \cdot(\theta, t)=\left(\theta+\phi \bmod \pi, a\left(t+w(\theta) \cdot A_{a}^{-1} R_{\phi}^{-1} b\right)\right),
$$

where $w(\theta)={ }^{t}(\cos \theta, \sin \theta)$, or equivalently

$$
(b, \phi, a)^{-1} \cdot(\theta, t)=\left(\theta-\phi \bmod \pi, \frac{t-w(\theta) \cdot b}{a}\right) .
$$

The isotropy at $\xi_{0}=(0,0)$ is

$$
H=\left\{\left(\left(0, b_{2}\right), \phi, a\right): b_{2} \in \mathbb{R}, \phi \in\{0, \pi\}, a \in \mathbb{R}^{+}\right\} .
$$

Thus, $[0, \pi) \times \mathbb{R}=S I M(2) / H$. An immediate calculation gives

$$
\int_{\Xi} f\left((b, \phi, a)^{-1} \cdot(\theta, t)\right) \mathrm{d} \theta \mathrm{d} t=a \int_{\Xi} f(\theta, t) \mathrm{d} \theta \mathrm{d} t, \quad f \in L^{1}(\Xi, \mathrm{d} \theta \mathrm{d} t),
$$

namely, (3) is satisfied with the character $\beta(b, \phi, a)=a$. Thus, the Lebesgue measure $d \xi=\mathrm{d} \theta \mathrm{d} t$ is a relatively invariant measure on $\Xi$. by

Consider now the sections $s: \mathbb{R}^{2} \rightarrow \operatorname{SIM}(2)$ and $\sigma:[0, \pi) \times \mathbb{R} \rightarrow S I M(2)$ defined

$$
s(x)=(x, 0,1), \quad \sigma(\theta, t)=(t w(\theta), \theta, 1) .
$$

It is easy to verify by direct computation that

$$
\begin{aligned}
& \hat{\xi}_{0}=H\left[x_{0}\right]=\left\{\left(0, b_{2}\right): b_{2} \in \mathbb{R}\right\} \simeq \mathbb{R}, \\
& \check{x}_{0}=K . \xi_{0}=\{(\theta, 0): \theta \in[0, \pi)\} \simeq[0, \pi) .
\end{aligned}
$$

It is immediate to see that the Lebesgue measure $\mathrm{d} b_{2}$ on $\hat{\xi}_{0}$ is a relatively $H$-invariant measure with character $\gamma\left(\left(0, b_{2}\right), \phi, a\right)=a$. Further, we have that

$$
\widehat{(\theta, t)}=\sigma(\theta, t)\left[\hat{\xi}_{0}\right]=\left\{x \in \mathbb{R}^{2}: x \cdot w(\theta)=t\right\},
$$

which is the set of all points laying on the line of equation $x \cdot w(\theta)=t$ and

$$
\check{x}=s(x) . \check{x}_{0}=\{(\theta, t) \in[0, \pi) \times \mathbb{R}: t-w(\theta) \cdot x=0\},
$$

which parametrizes the set of all lines passing through the point $x$.

It is easy to verify that $X=\mathbb{R}^{2}$ and $\Xi=[0, \pi) \times \mathbb{R}$ are homogeneous spaces in duality. Indeed, the map $x \mapsto \check{x}$ which sends a point to the set of all lines passing through that point and the map $(\theta, t) \mapsto \widehat{(\theta, t)}$ which sends a line to the set of points laying on that line are both injective. 


\subsection{The representations}

The group $G$ acts unitarily on $L^{2}(X, \mathrm{~d} x)$ via the quasi-regular representation defined by

$$
\pi(g) f(x)=|\operatorname{det}(k)|^{-1 / 2} f\left(k^{-1}(x-b)\right), \quad g=(b, k) .
$$

By Assumption (A5) $\pi$ is irreducible and square-integrable. We stress that this latter condition is needed only in Section 4 .

The group $G$ acts also on $L^{2}(\Xi, \mathrm{d} \xi)$ via the quasi-regular representation

$$
\hat{\pi}(g) F(\xi)=\beta(g)^{-1 / 2} F\left(g^{-1} \cdot \xi\right),
$$

where $\beta(g)$ is defined in (3). The representation $\hat{\pi}$ is irreducible by Assumption (A5) however this condition is not needed until Theorem 5 .

Example 1 (continued). The group $\operatorname{SIM}(2)$ acts on $L^{2}\left(\mathbb{R}^{2}\right)$ by means of the unitary irreducible representation $\pi$ defined by

$$
\pi(b, \phi, a) f(x)=a^{-1} f\left(A_{a}^{-1} R_{\phi}^{-1}(x-b)\right),
$$

or, equivalently, in the frequency domain

$$
\mathcal{F}[\pi(b, \phi, a) f](\xi)=a e^{-2 \pi i b \cdot \xi} \mathcal{F} f\left(A_{a} R_{\phi}^{-1} \xi\right) .
$$

As above, we consider on $\Xi=[0, \pi) \times \mathbb{R}$ the measure $\mathrm{d} \xi=\mathrm{d} \theta \mathrm{d} t$, where $\mathrm{d} \theta$ and $\mathrm{d} t$ are the Lebesgue measures on $[0, \pi)$ and $\mathbb{R}$ respectively. Then, since $\beta(b, \phi, a)=a, G$ acts on $L^{2}([0, \pi) \times \mathbb{R}, \mathrm{d} \theta \mathrm{d} t)$ by means of the quasi-regular representation $\hat{\pi}$ defined by

$$
\hat{\pi}(b, \phi, a) F(\theta, t)=a^{-\frac{1}{2}} F\left(\theta-\phi \bmod \pi, \frac{t-w(\theta) \cdot b}{a}\right),
$$

which is irreducible, too.

\subsection{The Radon transform}

Following Helgason's theory and using the Borel section $\sigma$ in order to push-forward the measure $\mathrm{d} m_{0}$ (see (4)) to the manifolds $\hat{\xi}$ given in (5), we define the Radon transform of $f$ as the map $\mathcal{R} f: \Xi \rightarrow \mathbb{C}$ given by

$$
\mathcal{R} f(\xi)=\int_{\hat{\xi}} f(x) \mathrm{d} m_{\xi}(x):=\int_{\hat{\xi}_{0}} f(\sigma(\xi)[x]) \mathrm{d} m_{0}(x) .
$$

Note that this depends intrinsically on the choices of $\mathrm{d} m_{0}$ and $\sigma$, and not only on the subset of integration $\hat{\xi}$.

Assumption (A7) states that there exists a non-trivial $\pi$-invariant subspace $\mathcal{A}$ of $L^{2}(X, \mathrm{~d} x)$ such that $\mathcal{R} f$ is well defined for all $f \in \mathcal{A}$ and the Radon transform $\mathcal{R}$ is a closable operator from $\mathcal{A}$ into $L^{2}(\Xi, \mathrm{d} \xi)$. We denote its closure by $\overline{\mathcal{R}}$. Clearly, $\mathcal{R} f$ may be well defined for a larger subset of functions than $\mathcal{A}$, however we will show that $\overline{\mathcal{R}}$ is independent of the choice of $\mathcal{A}$.

Example 1 (continued). We compute by (11) the Radon transform between the homogeneous spaces in duality $\mathbb{R}^{2}$ and $[0, \pi) \times \mathbb{R}$ and we obtain

$$
\mathcal{R}^{\mathrm{pol}} f(\theta, t)=\int_{\mathbb{R}} f(t \cos \theta-y \sin \theta, t \sin \theta+y \cos \theta) \mathrm{d} y,
$$


which is the so-called polar Radon transform.

Next we choose the domain $\mathcal{A}$ of $\mathcal{R}^{\text {pol }}$, which will be called $\mathcal{A}^{\text {pol }}$. This requires to recall one of the fundamental results in Radon theory, the so-called Fourier slice theorem [20, which relates the Radon transform with the Fourier transform. For $f \in L^{1}\left(\mathbb{R}^{2}\right)$, the integral (12) converges for almost all $(\theta, t) \in[0, \pi) \times \mathbb{R}$ by Fubini's theorem and

$$
(I \otimes \mathcal{F})\left(\mathcal{R}^{\mathrm{pol}} f\right)(\theta, \tau)=\mathcal{F} f(\tau w(\theta))
$$

for every $(\theta, \tau) \in[0, \pi) \times \mathbb{R}$, where $I$ is the identity operator. Hence, we have

$$
\begin{aligned}
\int_{[0, \pi) \times \mathbb{R}}\left|\mathcal{R}^{\mathrm{pol}} f(\theta, t)\right|^{2} \mathrm{~d} \theta \mathrm{d} t & =\int_{[0, \pi)} \int_{\mathbb{R}}\left|(I \otimes \mathcal{F})\left(\mathcal{R}^{\mathrm{pol}} f\right)(\theta, \tau)\right|^{2} \mathrm{~d} \tau \mathrm{d} \theta \\
& =\int_{[0, \pi) \times \mathbb{R}}|\mathcal{F} f(\tau w(\theta))|^{2} \mathrm{~d} \theta \mathrm{d} \tau \\
& =\int_{\mathbb{R}^{2}} \frac{\left|\mathcal{F} f\left(\xi_{1}, \xi_{2}\right)\right|^{2}}{\sqrt{\xi_{1}^{2}+\xi_{2}^{2}}} \mathrm{~d} \xi_{1} \mathrm{~d} \xi_{2} .
\end{aligned}
$$

We are thus led to choose

$$
\mathcal{A}^{\mathrm{pol}}=\left\{f \in L^{1}\left(\mathbb{R}^{2}\right) \cap L^{2}\left(\mathbb{R}^{2}\right): \int_{\mathbb{R}^{2}} \frac{\left|\mathcal{F} f\left(\xi_{1}, \xi_{2}\right)\right|^{2}}{\sqrt{\xi_{1}^{2}+\xi_{2}^{2}}} \mathrm{~d} \xi_{1} \mathrm{~d} \xi_{2}<+\infty\right\},
$$

which by (9) is $\pi$-invariant and by definition, $\mathcal{R}^{\text {pol }} f \in L^{2}([0, \pi) \times \mathbb{R})$ for all $f \in \mathcal{A}^{\text {pol }}$.

Now, we show that $\mathcal{R}^{\text {pol }}$ restricted to $\mathcal{A}^{\text {pol }}$ is closable. Suppose that $\left(f_{n}\right)_{n} \subset \mathcal{A}^{\mathrm{pol}}$ is a sequence such that $f_{n} \rightarrow f$ in $L^{2}\left(\mathbb{R}^{2}\right)$ and $\mathcal{R}^{\text {pol }} f_{n} \rightarrow g$ in $L^{2}([0, \pi) \times \mathbb{R})$. Since $I \otimes \mathcal{F}$ is unitary from $L^{2}([0, \pi) \times \mathbb{R})$ onto $L^{2}([0, \pi) \times \mathbb{R})$, we have that $(I \otimes \mathcal{F}) \mathcal{R}^{\text {pol }} f_{n} \rightarrow(I \otimes \mathcal{F}) g$ in $L^{2}([0, \pi) \times \mathbb{R})$. Since $f_{n} \in \mathcal{A}^{\text {pol }}$, by (13), for every $(\theta, \tau) \in[0, \pi) \times \mathbb{R}$

$$
(I \otimes \mathcal{F}) \mathcal{R}^{\mathrm{pol}} f_{n}(\theta, \tau)=\mathcal{F} f_{n}(\tau w(\theta)) .
$$

Hence, passing to a subsequence if necessary,

$$
\mathcal{F} f_{n}(\tau w(\theta)) \rightarrow(I \otimes \mathcal{F}) g(\theta, \tau)
$$

for almost every $(\theta, \tau) \in[0, \pi) \times \mathbb{R}$. Therefore, for almost every $(\theta, \tau) \in[0, \pi) \times \mathbb{R}$

$$
(I \otimes \mathcal{F}) g(\theta, \tau)=\lim _{n \rightarrow+\infty} \mathcal{F} f_{n}(\tau w(\theta))=\mathcal{F} f(\tau w(\theta)),
$$

where the last equality holds true using a subsequence if necessary. Therefore, if $\left(h_{n}\right)_{n} \in \mathcal{A}^{\text {pol }}$ is another sequence such that $h_{n} \rightarrow f$ in $L^{2}\left(\mathbb{R}^{2}\right)$ and $\mathcal{R}^{\text {pol }} h_{n} \rightarrow h$ in $L^{2}([0, \pi) \times \mathbb{R})$, then, for almost every $(\theta, \tau) \in[0, \pi) \times \mathbb{R}$

$$
(I \otimes \mathcal{F}) h(\theta, \tau)=\mathcal{F} f(\tau w(\theta)) .
$$

Therefore,

$$
(I \otimes \mathcal{F}) g(\theta, \tau)=(I \otimes \mathcal{F}) h(\theta, \tau)
$$

for almost every $(\theta, \tau) \in[0, \pi) \times \mathbb{R}$. Then $\lim _{n \rightarrow+\infty} \mathcal{R}^{\mathrm{pol}} f_{n}=\lim _{n \rightarrow+\infty} \mathcal{R}^{\mathrm{pol}} h_{n}$, and $\mathcal{R}^{\mathrm{pol}}$ is closable. 


\section{The Unitarization Theorem}

Our construction is based on the following lemma, which shows that the Radon transform intertwines the representations $\pi$ and $\hat{\pi}$ up to a positive character of $G$. For the classical Radon transform considered in Example 1, this result is a direct consequence of the behavior of $\mathcal{R}^{\mathrm{pol}}$ under linear actions [29, Chapter 2].

Lemma 2. The Radon transform $\mathcal{R}$ restricted to $\mathcal{A}$ is a densely defined operator from $\mathcal{A}$ into $L^{2}(\Xi, \mathrm{d} \xi)$ satisfying

$$
\mathcal{R} \pi(g)=\chi(g)^{-1} \hat{\pi}(g) \mathcal{R},
$$

for all $g \in G$, where

$$
\chi(g)=\beta(g)^{-1 / 2}|\operatorname{det}(k)|^{1 / 2} \gamma\left(g \sigma\left(g^{-1} \cdot \xi_{0}\right)\right)^{-1}, \quad g=(b, k) \in G .
$$

With a slight abuse of notation, $\mathcal{R}$ denotes both the Radon transform defined by (11) and its restriction to $\mathcal{A}$.

Proof. By Assumption (A7) $\mathcal{R}$ is a well-defined operator from $\mathcal{A}$ into $L^{2}(\Xi, \mathrm{d} \xi)$. We now prove (14). By the $\pi$-invariance of $\mathcal{A}$, for $f \in \mathcal{A}$ and $g=(b, k) \in G$ we have

$$
\begin{aligned}
(\mathcal{R} \pi(b, k) f)(\xi) & =|\operatorname{det}(k)|^{-1 / 2} \int_{\hat{\xi}_{0}} f\left((b, k)^{-1} \sigma(\xi)[x]\right) \mathrm{d} m_{0}(x) \\
& =|\operatorname{det}(k)|^{-1 / 2} \int_{\hat{\xi}_{0}} f\left(\sigma\left((b, k)^{-1} \cdot \xi\right) \sigma\left((b, k)^{-1} \cdot \xi\right)^{-1}(b, k)^{-1} \sigma(\xi)[x]\right) \mathrm{d} m_{0}(x) \\
& =|\operatorname{det}(k)|^{-1 / 2} \int_{\hat{\xi}_{0}} f\left(\sigma\left((b, k)^{-1} \cdot \xi\right) m((b, k), \xi)^{-1}[x]\right) \mathrm{d} m_{0}(x),
\end{aligned}
$$

where $m((b, k), \xi)^{-1}:=\sigma\left((b, k)^{-1} \cdot \xi\right)^{-1}(b, k)^{-1} \sigma(\xi)$. It is known that for any $g \in G$ and any $\xi \in \Xi$

$$
m(g, \xi)=\sigma(\xi)^{-1} g \sigma\left(g^{-1} \cdot \xi\right) \in H .
$$

We show this property for the reader's convenience. Indeed

$$
\sigma(\xi)^{-1} g \sigma\left(g^{-1} \cdot \xi\right) \cdot \xi_{0}=\sigma(\xi)^{-1} g \cdot\left(g^{-1} \cdot \xi\right)=\sigma(\xi)^{-1} \cdot \xi=\xi_{0},
$$

so that $m(g, \xi) \in H$. Thus, using (4) we obtain

$$
(\mathcal{R} \pi(b, k) f)(\xi)=|\operatorname{det}(k)|^{-1 / 2} \gamma(m((b, k), \xi)) \int_{\hat{\xi}_{0}} f\left(\sigma\left((b, k)^{-1} \cdot \xi\right)[x]\right) \mathrm{d} m_{0}(x) .
$$

Then,

$$
\begin{aligned}
(\mathcal{R} \pi(b, k) f)(\xi) & =|\operatorname{det}(k)|^{-1 / 2} \gamma(m((b, k), \xi))(\mathcal{R} f)\left((b, k)^{-1} \cdot \xi\right) \\
& =\beta(b, k)^{1 / 2}|\operatorname{det}(k)|^{-1 / 2} \gamma(m((b, k), \xi)) \hat{\pi}(b, k) \mathcal{R} f(\xi) .
\end{aligned}
$$

Thanks to assumption (A3), $(g, \xi) \mapsto \gamma(m(g, \xi))$ extends to a positive character of $G$ independent of $\xi$. In particular, $\gamma(m(g, \xi))=\gamma\left(m\left(g, \xi_{0}\right)\right)=\gamma\left(g \sigma\left(g^{-1} \cdot \xi_{0}\right)\right)$, and (14) follows.

We finally prove that $\mathcal{A}$ is dense. By assumption (A7) the domain of $\mathcal{R}$ is $\pi$ invariant, so that $\pi(g) \overline{\mathcal{A}} \subset \overline{\mathcal{A}}$ for every $g \in G$. Since $\overline{\mathcal{A}} \neq\{0\}$ and $\pi$ is irreducible, then $\overline{\mathcal{A}}=L^{2}(X, \mathrm{~d} x)$. 
Observe that, if $\gamma$ extends to a positive character of $G$, then

$$
\gamma(m(g, \xi))=\gamma(\sigma(\xi))^{-1} \gamma(g) \gamma\left(\sigma\left(g^{-1} \cdot \xi\right)\right)
$$

and the independence of $\xi$ is implied by the stronger condition

$$
\gamma\left(\sigma\left(g^{-1} \cdot \xi\right)\right)=\gamma(\sigma(\xi)),
$$

that must be satisfied for all $g \in G$ and $\xi \in \Xi$. This is equivalent to requiring that $\gamma(\sigma(\xi))=1$ for all $\xi \in \Xi$, which is true in all our examples.

Example 1 (continued). By (14) and (15) we have that

$$
\mathcal{R}^{\mathrm{pol}} \pi(b, \phi, a)=\chi(b, \phi, a)^{-1} \hat{\pi}(b, \phi, a) \mathcal{R}^{\mathrm{pol}},
$$

where $\chi(b, \phi, a)=a^{-1 / 2}$ is a character of $G$, since $\beta(b, \phi, a)=\gamma(b, \phi, a)=a$ and $\operatorname{det}\left(R_{\phi} A_{a}\right)=a^{2}$.

The following result is the key of our construction. Recall that by Assumption (A7) the operator $\mathcal{R}: \mathcal{A} \subseteq L^{2}(X, \mathrm{~d} x) \rightarrow L^{2}(\Xi, \mathrm{d} \xi)$ is closable.

Lemma 3. The closure $\overline{\mathcal{R}}$ of the Radon transform $\mathcal{R}$ is a densely defined operator satisfying

$$
\overline{\mathcal{R}} \pi(g)=\chi(g)^{-1} \hat{\pi}(g) \overline{\mathcal{R}},
$$

for all $g \in G$, where $\chi$ is given by (15).

Proof. Since $\mathcal{R}$ is densely defined by Lemma 2 , its closure $\overline{\mathcal{R}}$ is a densely defined operator from $L^{2}(X, \mathrm{~d} x)$ to $L^{2}(\Xi, \mathrm{d} \xi)$. Now, we prove that $\overline{\mathcal{R}}$ satisfies (18). We start by proving that the domain of $\overline{\mathcal{R}}$ is $\pi$-invariant. Given $f \in \operatorname{dom}(\overline{\mathcal{R}})$, let $\left(f_{n}\right)_{n} \in \mathcal{A}$ be a sequence such that $f_{n} \rightarrow f$ in $L^{2}(X, \mathrm{~d} x)$ and $\mathcal{R} f_{n} \rightarrow \overline{\mathcal{R}} f$ in $L^{2}(\Xi, \mathrm{d} \xi)$. In order to prove that $\pi(g) f \in \operatorname{dom}(\overline{\mathcal{R}})$ it is enough to show that the sequence $\left(\mathcal{R} \pi(g) f_{n}\right)_{n}$ converges in $L^{2}(\Xi, \mathrm{d} \xi)$ : indeed $\pi(g) f_{n} \in \mathcal{A}$ for any $n \in \mathbb{N}$ because $\mathcal{A}$ is $\pi$-invariant and $\pi(g) f_{n} \rightarrow \pi(g) f$ in $L^{2}(X, \mathrm{~d} x)$ because $\pi(g)$ is a unitary operator for any $g \in G$. But then, by property (14) we have that

$$
\begin{aligned}
\lim _{n \rightarrow+\infty} \mathcal{R} \pi(g) f_{n} & =\chi(g)^{-1} \lim _{n \rightarrow+\infty} \hat{\pi}(g) \mathcal{R} f_{n} \\
& =\chi(g)^{-1} \hat{\pi}(g) \lim _{n \rightarrow+\infty} \mathcal{R} f_{n} \\
& =\chi(g)^{-1} \hat{\pi}(g) \overline{\mathcal{R}} f,
\end{aligned}
$$

so that $\left(\mathcal{R} \pi(g) f_{n}\right)_{n}$ converges in $L^{2}(\Xi, \mathrm{d} \xi)$. Then, $\pi(g) f \in \operatorname{dom}(\overline{\mathcal{R}})$ and by definition $\mathcal{R} \pi(g) f_{n} \rightarrow \overline{\mathcal{R}} \pi(g) f$. Therefore, by (19), $\overline{\mathcal{R}}$ satisfies (18).

Our main theorem is based on the following classical result due to Duflo and Moore 12. According to [12, a densely defined closed operator $T$ from a Hilbert space $\mathcal{H}$ to another Hilbert space $\hat{\mathcal{H}}$ is called semi-invariant with weight $\zeta$ if it satisfies

$$
\hat{\pi}(g) T \pi(g)^{-1}=\zeta(g) T, \quad g \in G,
$$

where $\zeta$ is a character of $G$ and $\pi$ and $\hat{\pi}$ are unitary representations of $G$ acting on $\mathcal{H}$ and $\hat{\mathcal{H}}$, respectively. 
Theorem 4 ([12, Theorem 1]). With the above notation, assume that $\pi$ is irreducible. Let $T$ be a densely defined closed nonzero operator from $\mathcal{H}$ to $\hat{\mathcal{H}}$, semi-invariant with weight $\zeta$.

(i) Suppose that $\pi=\hat{\pi}$. Let $T^{\prime}$ be another densely defined closed operator from $\mathcal{H}$ to $\mathcal{H}$, semi-invariant with weight $\zeta$. Then $T^{\prime}$ is proportional to $T$.

(ii) Let $T=\mathcal{Q}|T|$ be the polar decomposition of $T$. Then $|T|$ is a positive selfadjoint operator in $\mathcal{H}$ semi-invariant with weight $|\zeta|$, and $\mathcal{Q}$ is a partial isometry of $\mathcal{H}$ into $\hat{\mathcal{H}}$, semi-invariant with weight $\zeta /|\zeta|$.

By observing that Lemma 3 shows that the Radon transform $\overline{\mathcal{R}}$ is a semi-invariant operator with weight given by (15), we are finally in a position to state and prove our main result. We stress that its proof does not use the transversality condition on $(X, \Xi)$ (assumption (A4), cfr. Remark 10) and the square-integrability of $\pi$; the irreducibility of $\hat{\pi}$ is only needed in the last claim of the theorem.

Theorem 5. There exists a unique positive self-adjoint operator

$$
\mathcal{I}: \operatorname{dom}(\mathcal{I}) \supseteq \operatorname{Im} \overline{\mathcal{R}} \rightarrow L^{2}(\Xi, \mathrm{d} \xi),
$$

semi-invariant with weight $\zeta=\chi^{-1}$ with the property that the composite operator $\mathcal{I} \overline{\mathcal{R}}$ extends to an isometry $\mathcal{Q}: L^{2}(X, \mathrm{~d} x) \rightarrow L^{2}(\Xi, \mathrm{d} \xi)$ intertwining $\pi$ and $\hat{\pi}$, namely

$$
\hat{\pi}(g) \mathcal{Q} \pi(g)^{-1}=\mathcal{Q}, \quad g \in G .
$$

Furthermore, if $\hat{\pi}$ is irreducible, then $\mathcal{Q}$ is a unitary operator and $\pi$ and $\hat{\pi}$ are equivalent representations.

The above result is a generalization of Helgason's theorem on the unitarization of the classical Radon transform, [20, Theorem 4.1], because by definition of extension it holds that

$$
\mathcal{I R} f=\mathcal{Q} f, \quad f \in \mathcal{A} .
$$

Proof. The unitarization of $\mathcal{R}$ is based on the polar decomposition $\overline{\mathcal{R}}=\mathcal{Q}|\overline{\mathcal{R}}|$ of $\overline{\mathcal{R}}$. By Lemma 3 and Theorem 4, item (ii), $|\overline{\mathcal{R}}|: \operatorname{dom}(\overline{\mathcal{R}}) \rightarrow L^{2}(X, \mathrm{~d} x)$ is a positive self-adjoint operator semi-invariant with weight $|\chi|=\chi$, where $\chi$ is defined by (15), i.e.

$$
\pi(g)|\overline{\mathcal{R}}| \pi(g)^{-1}=\chi(g)|\overline{\mathcal{R}}|, \quad g \in G,
$$

and $\mathcal{Q}: L^{2}(X, \mathrm{~d} x) \rightarrow L^{2}(\Xi, \mathrm{d} \xi)$ is a partial isometry with

$$
\operatorname{ker} \mathcal{Q}=\operatorname{ker} \overline{\mathcal{R}}, \quad \operatorname{Im} \mathcal{Q}=\overline{\operatorname{Im}(\overline{\mathcal{R}})},
$$

and is semi-invariant with weight $\chi /|\chi| \equiv 1$, i.e. (21) is satisfied. Since $\pi$ is irreducible, ker $\mathcal{Q}=\{0\}$ and it follows that $\mathcal{Q}$ is an isometry.

Define $W=\mathcal{Q}|\overline{\mathcal{R}}| \mathcal{Q}^{*}$ with $\hat{\pi}$-invariant domain

$$
\operatorname{dom} W=\left\{f \in L^{2}(\Xi, \mathrm{d} \xi): \mathcal{Q}^{*} f \in \operatorname{dom} \overline{\mathcal{R}}\right\}=\mathcal{Q}(\operatorname{dom} \overline{\mathcal{R}}) \oplus \overline{\operatorname{Im}(\overline{\mathcal{R}})}^{\perp},
$$

which is a densely defined positive operator in $L^{2}(\Xi, \mathrm{d} \xi)$, semi-invariant with weight $\chi$. Indeed, $\mathcal{Q}(\operatorname{dom} \overline{\mathcal{R}})$ is dense in $\mathcal{Q}\left(L^{2}(X, \mathrm{~d} x)\right)=\overline{\operatorname{Im}(\overline{\mathcal{R}})}$ since $\overline{\mathcal{R}}$ is densely defined by Lemma 3. Observe that the $\hat{\pi}$-invariance of $\operatorname{dom} W$ follows from the $\pi$-invariance of 
dom $\overline{\mathcal{R}}$. Further, by (21) and (23) and using that $\pi(g)$ is a unitary operator we readily derive

$$
\begin{aligned}
\hat{\pi}(g) W \hat{\pi}(g)^{-1} f & =\hat{\pi}(g) \mathcal{Q}|\overline{\mathcal{R}}| \mathcal{Q}^{*} \hat{\pi}(g)^{-1} f \\
& =\left(\hat{\pi}(g) \mathcal{Q} \pi(g)^{-1}\right)\left(\pi(g)|\overline{\mathcal{R}}| \pi(g)^{-1}\right)\left(\pi(g) \mathcal{Q}^{*} \hat{\pi}(g)^{-1}\right) f \\
& =\mathcal{Q}(\chi(g)|\overline{\mathcal{R}}|) \mathcal{Q}^{*} f \\
& =\chi(g) W f
\end{aligned}
$$

for every $f \in \operatorname{dom} W$.

Since $\mathcal{Q}^{*} \mathcal{Q}=\operatorname{Id}$, then $\overline{\mathcal{R}}=W \mathcal{Q}$ and $\operatorname{Im} \overline{\mathcal{R}} \subset \operatorname{Im} W$. We denote by $\mathcal{I}$ the MoorePenrose inverse of $W[\underline{6}$, Chapter $9, \S 3$, Theorem 2] with densely defined domain given by

$$
\operatorname{Im} W \oplus \operatorname{Im} W^{\perp} \supset \operatorname{Im} W \mathcal{Q}=\operatorname{Im} \overline{\mathcal{R}} .
$$

Since $W$ is a positive operator in $L^{2}(\Xi, \mathrm{d} \xi)$, then $\mathcal{I}$ is positive, too, and

$$
\begin{array}{ll}
\mathcal{I} W f=f, & f \in \operatorname{dom} W \cap \operatorname{ker} W^{\perp}, \\
W \mathcal{I} f=f, & f \in \operatorname{Im} W .
\end{array}
$$

We claim that $\mathcal{I}$ is semi-invariant with weight $\chi^{-1}$ and

$$
\mathcal{I} \overline{\mathcal{R}} f=\mathcal{Q} f, \quad f \in \operatorname{dom} \overline{\mathcal{R}} .
$$

Indeed, if $f \in \operatorname{Im} W$, by definition $\mathcal{I} f=h$ with $h \in \operatorname{dom} W \cap \operatorname{ker} W^{\perp}$ and $W h=f$. Thus, by the semi-invariance of $W$ we have that

$$
\begin{aligned}
\hat{\pi}(g) \mathcal{I} \hat{\pi}(g)^{-1} f & =\hat{\pi}(g) \mathcal{I} \hat{\pi}(g)^{-1} W h \\
& =\chi(g)^{-1} \hat{\pi}(g) \mathcal{I} W \hat{\pi}(g)^{-1} h \\
& =\chi(g)^{-1} \mathcal{I} f,
\end{aligned}
$$

where we used that $\hat{\pi}(g)^{-1} h \in \operatorname{ker} W^{\perp}$, which follows from the $\hat{\pi}$-invariance of ker $W$. If $f \in \operatorname{Im} W^{\perp}$, by definition of $\mathcal{I}$ the semi-invariance property (24) is trivial.

Finally, since by (18) $\overline{\mathcal{R}}$ is an injective operator, we have that $\operatorname{ker} W=\operatorname{ker} \mathcal{Q}^{*}$ and hence ker $W^{\perp}=\overline{\operatorname{Im} \mathcal{Q}} \supset \operatorname{Im} \mathcal{Q}$, whence $\mathcal{Q} f \in \operatorname{dom} W \cap \operatorname{ker} W^{\perp}$ for any $f \in \operatorname{dom} \overline{\mathcal{R}}$. Therefore $\mathcal{I} \overline{\mathcal{R}} f=\mathcal{I} W \mathcal{Q} f=\mathcal{Q} f$, as desired.

Assume now that $\hat{\pi}$ is irreducible. Since $\operatorname{Im}(\mathcal{Q})$ is a $\hat{\pi}$-invariant closed subspace of $L^{2}(\Xi, \mathrm{d} \xi)$ by (21) , then $\mathcal{Q}$ is surjective, so that $\mathcal{Q}$ is unitary and $\pi$ and $\hat{\pi}$ are equivalent by (21).

It is worth observing that the results of this section do not depend on the choice of the subspace $\mathcal{A}$. Indeed, suppose to have another $\pi$-invariant subspace

$$
\mathcal{A}^{\prime} \subseteq\left\{f \in L^{2}(X, \mathrm{~d} x): f(\sigma(\xi)[\cdot]) \in L^{1}\left(\hat{\xi}_{0}, m_{0}\right) \text { a.e. } \xi \in \Xi, \mathcal{R} f \in L^{2}(\Xi, \mathrm{d} \xi)\right\}
$$

of $L^{2}(X, \mathrm{~d} x), \mathcal{A}^{\prime} \neq\{0\}$, such that $\mathcal{R}$ restricted to $\mathcal{A}^{\prime}$ is a closable operator. We denote by $\overline{\mathcal{R}^{\prime}}$ the closure of the restriction of $\mathcal{R}$ to $\mathcal{A}^{\prime}$. We prove that $\overline{\mathcal{R}^{\prime}}$ actually coincides with $\overline{\mathcal{R}}$. By Lemma 3 the closure $\overline{\mathcal{R}^{\prime}}$ is a densely defined operator satisfying

$$
\overline{\mathcal{R}^{\prime}} \pi(g) f=\chi(g)^{-1} \hat{\pi}(g) \overline{\mathcal{R}^{\prime}} f, \quad f \in \operatorname{dom}\left(\overline{\mathcal{R}^{\prime}}\right) .
$$

Thus, by Theorem 4 , letting $\overline{\mathcal{R}^{\prime}}=\mathcal{Q}^{\prime}\left|\overline{\mathcal{R}^{\prime}}\right|$ be the polar decomposition of $\overline{\mathcal{R}^{\prime}}$, we have that $\left|\overline{\mathcal{R}^{\prime}}\right|: \operatorname{dom}\left(\overline{\mathcal{R}^{\prime}}\right) \rightarrow L^{2}(X, \mathrm{~d} x)$ is a positive selfadjoint operator semi-invariant with weight $|\chi|=\chi$ defined by (15). Therefore, $\left|\overline{\mathcal{R}^{\prime}}\right|$ and $|\overline{\mathcal{R}}|$ are proportional by Theorem 4 , item (i). This implies that their domains, $\operatorname{dom}\left(\overline{\mathcal{R}^{\prime}}\right)$ and $\operatorname{dom}(\overline{\mathcal{R}})$, coincide. Hence, $\overline{\mathcal{R}^{\prime}}=\overline{\mathcal{R}}$. 
Example 1 (continued). Applying Lemma 3 to $\mathcal{R}^{\text {pol }}$, by 117 its closure $\overline{\mathcal{R}^{\text {pol }}}$ is a semi-invariant operator from $\mathcal{A}^{\text {pol }}$ to $L^{2}([0, \pi) \times \mathbb{R})$ with weight $\chi(b, \phi, a)=a^{-1 / 2}$. By Theorem 5 there exists a positive selfadjoint operator $\mathcal{I}: \operatorname{dom}(\mathcal{I}) \supseteq \operatorname{Im}\left(\overline{\mathcal{R}^{\text {pol }}}\right) \rightarrow$ $L^{2}([0, \pi) \times \mathbb{R})$, semi-invariant with weight $\chi(g)^{-1}=a^{1 / 2}$, such that $\overline{\mathcal{I}} \overline{\mathcal{R}}^{\text {pol }}$ extends to a unitary operator $\mathcal{Q}: L^{2}\left(\mathbb{R}^{2}\right) \rightarrow L^{2}([0, \pi) \times \mathbb{R})$ intertwining the quasi-regular (irreducible) representations $\pi$ and $\hat{\pi}$. Hence

$$
\begin{array}{ll}
\mathcal{I R}^{\mathrm{pol}} f=\mathcal{Q} f & f \in \mathcal{A}^{\mathrm{pol}}, \\
\mathcal{Q}^{*} \mathcal{Q} f=f & f \in L^{2}\left(\mathbb{R}^{2}\right), \\
\mathcal{Q Q}^{*} F=F & F \in L^{2}([0, \pi) \times \mathbb{R}), \\
\hat{\pi}(g) \mathcal{Q} \pi(g)^{-1}=\mathcal{Q} & g \in \operatorname{SIM}(2) .
\end{array}
$$

We can provide an explicit formula for $\mathcal{I}$. Consider the subspace

$$
\mathcal{D}=\left\{f \in L^{2}([0, \pi) \times \mathbb{R}): \int_{[0, \pi) \times \mathbb{R}}|\tau||(I \otimes \mathcal{F}) f(\theta, \tau)|^{2} \mathrm{~d} \theta \mathrm{d} \tau<+\infty\right\}
$$

and define the operator $\mathcal{J}: \mathcal{D} \rightarrow L^{2}([0, \pi) \times \mathbb{R})$ by

$$
(I \otimes \mathcal{F}) \mathcal{J} f(\theta, \tau)=|\tau|^{\frac{1}{2}}(I \otimes \mathcal{F}) f(\theta, \tau)
$$

a Fourier multiplier with respect to the last variable. A direct calculation shows that $\mathcal{J}$ is a densely defined positive self-adjoint injective operator and is semi-invariant with weight $\zeta(g)=\chi(g)^{-1}=a^{1 / 2}$. By Theorem 4 , item (i), there exists $c>0$ such that $\mathcal{I}=c \mathcal{J}$ and we now show that $c=1$. Consider a function $f \in \mathcal{A}^{\text {pol }} \backslash\{0\}$. Then, by Plancherel theorem and the Fourier slice theorem (13) we have that

$$
\begin{aligned}
\|f\|^{2}=\left\|\mathcal{I} \mathcal{R}^{\mathrm{pol}} f\right\|_{L^{2}([0, \pi) \times \mathbb{R})}^{2} & =c^{2}\left\|(I \otimes \mathcal{F}) \mathcal{J} \mathcal{R}^{\mathrm{pol}} f\right\|_{L^{2}([0, \pi) \times \mathbb{R})}^{2} \\
& =c^{2} \int_{[0, \pi) \times \mathbb{R}}\left|\tau \|(I \otimes \mathcal{F}) \mathcal{R}^{\mathrm{pol}} f(\theta, \tau)\right|^{2} \mathrm{~d} \theta \mathrm{d} \tau \\
& =c^{2} \int_{[0, \pi) \times \mathbb{R}}|\tau \| \mathcal{F} f(\tau w(\theta))|^{2} \mathrm{~d} \theta \mathrm{d} \tau \\
& =c^{2}\|f\|^{2} .
\end{aligned}
$$

Thus, we obtain $c=1$.

\section{Inversion of the Radon transform}

In this section, we make explicit use of the assumption that $\pi$ is square-integrable to invert the Radon transform. We recall that, under this assumption, there exists a self-adjoint operator

$$
C: \operatorname{dom} C \subseteq L^{2}(X, \mathrm{~d} x) \rightarrow L^{2}(X, \mathrm{~d} x),
$$

semi-invariant with weight $\Delta^{\frac{1}{2}}$, where $\Delta$ is the modular function of $G$, such that for all $\psi \in \operatorname{dom} C$ with $\|C \psi\|=1$, the voice transform $\mathcal{V}_{\psi}$

$$
\left(\mathcal{V}_{\psi} f\right)(g)=\langle f, \pi(g) \psi\rangle, \quad g \in G,
$$


is an isometry from $L^{2}(X, \mathrm{~d} x)$ into $L^{2}(G)$ and we have the weakly-convergent reproducing formula

$$
f=\int_{G}\left(\mathcal{V}_{\psi} f\right)(g) \pi(g) \psi \mathrm{d} \mu(g),
$$

where $\mu$ is the Haar measure (see, for example, [15, Theorem 2.25]). The vector $\psi$ is called admissible vector.

As shown in the previous section, there exists a positive self-adjoint operator $\mathcal{I}$ semi-invariant with weight $\chi^{-1}$ such that $\mathcal{I R}$ extends to a unitary operator $\mathcal{Q}$, which intertwines the quasi-regular representations $\pi$ and $\hat{\pi}$ of $G$ on $L^{2}(X, \mathrm{~d} x)$ and $L^{2}(\Xi, \mathrm{d} \xi)$ respectively.

Since $\mathcal{Q}$ is unitary and satisfies (21), the voice transform reads

$$
\mathcal{V}_{\psi} f(g)=\langle f, \pi(g) \psi\rangle=\langle\mathcal{Q} f, \mathcal{Q} \pi(g) \psi\rangle=\langle\mathcal{Q} f, \hat{\pi}(g) \mathcal{Q} \psi\rangle, \quad g \in G,
$$

for all $f \in L^{2}(X, \mathrm{~d} x)$. Furthermore, the assumption that $\pi$ is square-integrable ensures that any $f \in L^{2}(X, \mathrm{~d} x)$ can be reconstructed from its unitary Radon transform $\mathcal{Q} f$ by means of the reconstruction formula (28), which becomes

$$
f=\int_{G}\langle\mathcal{Q} f, \hat{\pi}(g) \mathcal{Q} \psi\rangle \pi(g) \psi \mathrm{d} \mu(g) .
$$

Moreover, if we can choose $\psi$ in such a way that $\mathcal{Q} \psi$ is in the domain of the operator $\mathcal{I}$, by (29), for all $f \in \operatorname{dom} \overline{\mathcal{R}}$, we have

$$
\begin{aligned}
\mathcal{V}_{\psi} f(g) & =\langle\mathcal{Q} f, \hat{\pi}(g) \mathcal{Q} \psi\rangle \\
& =\langle\mathcal{I} \overline{\mathcal{R}} f, \hat{\pi}(g) \mathcal{Q} \psi\rangle \\
& =\langle\overline{\mathcal{R}} f, \mathcal{I} \hat{\pi}(g) \mathcal{Q} \psi\rangle \\
& =\chi(g)\langle\overline{\mathcal{R}} f, \hat{\pi}(g) \mathcal{I} \mathcal{Q} \psi\rangle
\end{aligned}
$$

where we use that $\mathcal{I}$ is a selfadjoint operator, semi-invariant with weight $\chi^{-1}$.

By (30) the voice transform $\mathcal{V}_{\psi} f$ depends on $f$ only through its Radon transform $\overline{\mathcal{R}} f$. Therefore, (30) together with (28) allow to reconstruct an unknown signal $f \in$ dom $\overline{\mathcal{R}}$ from its Radon transform. Explicitly, we have derived the following inversion formula for the Radon transform.

Theorem 6. Let $\psi \in L^{2}(X, \mathrm{~d} x)$ be an admissible vector for $\pi$ such that $\mathcal{Q} \psi \in \operatorname{dom} \mathcal{I}$, and set $\Psi=\mathcal{I} \mathcal{Q} \psi$. Then, for any $f \in \operatorname{dom} \overline{\mathcal{R}}$,

$$
f=\int_{G} \chi(g)\langle\overline{\mathcal{R}} f, \hat{\pi}(g) \Psi\rangle \pi(g) \psi \mathrm{d} \mu(g),
$$

where the integral is weakly convergent, and

$$
\|f\|^{2}=\int_{G} \chi(g)^{2}|\langle\overline{\mathcal{R}} f, \hat{\pi}(g) \Psi\rangle|^{2} \mathrm{~d} \mu(g) .
$$

If, in addition, $\psi \in \operatorname{dom} \overline{\mathcal{R}}$, then $\Psi=\mathcal{I}^{2} \overline{\mathcal{R}} \psi$.

Note that the datum $\overline{\mathcal{R}} f$ is analyzed by the family $\{\hat{\pi}(g) \Psi\}_{g \in G}$ and the signal $f$ is reconstructed by a different family, namely $\{\pi(g) \psi\}_{g \in G}$. 
Example1 (continued). It is known that $\pi$ is square-integrable and the corresponding voice transform gives rise to $2 D$-directional wavelets [4. An admissible vector is a function $\psi \in L^{2}\left(\mathbb{R}^{2}\right)$ satisfying the following admissibility condition [4]

$$
\int_{[0,2 \pi) \times \mathbb{R}^{+}}\left|\mathcal{F} \psi\left(A_{a} R_{\phi}^{-1} \xi\right)\right|^{2} \mathrm{~d} \phi \frac{\mathrm{d} a}{a}=1, \quad \text { for all } \xi \in \mathbb{R}^{2} /\{0\},
$$

which is equivalent to

$$
\int_{\mathbb{R}^{2}} \frac{\left|\mathcal{F} \psi\left(\xi_{1}, \xi_{2}\right)\right|^{2}}{\xi_{1}^{2}+\xi_{2}^{2}} \mathrm{~d} \xi_{1} \mathrm{~d} \xi_{2}=1
$$

Given $f \in \mathcal{A}^{\text {pol }}$, define $\mathcal{G}(b, \phi, a)=a^{\frac{1}{2}}\left\langle\mathcal{R}^{\text {pol }} f, \hat{\pi}(b, \phi, a) \Psi\right\rangle$, i.e. by (10)

$$
\mathcal{G}(b, \phi, a)=\int_{[0, \pi) \times \mathbb{R}} \mathcal{R}^{\mathrm{pol}} f(\theta, t) \overline{\Psi\left(\theta-\phi \bmod \pi, \frac{t-b \cdot w(\theta)}{a}\right)} \mathrm{d} \theta \mathrm{d} t .
$$

Then, taking into account that $\chi(b, \phi, a)=a^{-\frac{1}{2}}$, (31) reads

$$
f(x)=\int_{\mathbb{R}^{2} \rtimes\left([0,2 \pi) \times \mathbb{R}^{+}\right)} \mathcal{G}(b, \phi, a) \psi\left(R_{\phi}^{-1} \frac{x-b}{a}\right) \mathrm{d} b \mathrm{~d} \phi \frac{\mathrm{d} a}{a^{5}} .
$$

By (32), reconstruction formula (35) is equivalent to

$$
\|f\|^{2}=\int_{\mathbb{R}^{2} \rtimes\left([0,2 \pi) \times \mathbb{R}^{+}\right)}|\mathcal{G}(b, \phi, a)|^{2} \mathrm{~d} b \mathrm{~d} \phi \frac{\mathrm{d} a}{a^{5}} .
$$

The idea to exploit the theory of the continuous wavelet transform to derive inversion formulae for the Radon transform is not new, we refer to [24, 7, 27, 31, 28]-to name a few.

It is possible to obtain a version of (36) in which the scale parameter $a$ varies only in a compact set. Consider a smooth function $\Phi \in L^{1}\left(\mathbb{R}^{2}\right) \cap L^{2}\left(\mathbb{R}^{2}\right)$ such that

$$
|\mathcal{F} \Phi(\xi)|^{2}+\int_{[0,2 \pi) \times(0,1)}\left|\mathcal{F} \psi\left(A_{a} R_{\phi}^{-1} \xi\right)\right|^{2} \mathrm{~d} \phi \frac{\mathrm{d} a}{a}=1 .
$$

By Plancherel theorem, we have that

$$
\begin{aligned}
\int_{\mathbb{R}^{2}}\left|\left\langle f, T_{b} \Phi\right\rangle\right|^{2} \mathrm{~d} b & =\int_{\mathbb{R}^{2}}\left|\int_{\mathbb{R}^{2}} \mathcal{F} f(\xi) \overline{\mathcal{F} \Phi(\xi)} e^{2 \pi i b \cdot \xi} \mathrm{d} \xi\right|^{2} \mathrm{~d} b \\
& =\int_{\mathbb{R}^{2}}\left|\mathcal{F}^{-1}(\mathcal{F} f \overline{\mathcal{F} \Phi})(b)\right|^{2} \mathrm{~d} b \\
& =\int_{\mathbb{R}^{2}}|\mathcal{F} f(\xi)|^{2}|\mathcal{F} \Phi(\xi)|^{2} \mathrm{~d} \xi
\end{aligned}
$$

Using an analogous computation, by Plancherel theorem, equation (9) and Fubini's 
theorem we have

$$
\begin{aligned}
& \int_{\mathbb{R}^{2} \rtimes([0,2 \pi) \times(0,1))}|\mathcal{G}(b, \phi, a)|^{2} \mathrm{~d} b \mathrm{~d} \phi \frac{\mathrm{d} a}{a^{5}}=\int_{\mathbb{R}^{2} \rtimes([0,2 \pi) \times(0,1))}|\langle f, \pi(b, \phi, a) \psi\rangle|^{2} \mathrm{~d} b \mathrm{~d} \phi \frac{\mathrm{d} a}{a^{3}} \\
& =\int_{\mathbb{R}^{2} \rtimes([0,2 \pi) \times(0,1))}\left|\int_{\mathbb{R}^{2}} \mathcal{F} f(\xi) \overline{\mathcal{F} \psi\left(A_{a} R_{\phi}^{-1} \xi\right)} e^{2 \pi i b \cdot \xi} \mathrm{d} \xi\right|^{2} \mathrm{~d} b \mathrm{~d} \phi \frac{\mathrm{d} a}{a} \\
& =\int_{[0,2 \pi) \times(0,1)}\left(\int_{\mathbb{R}^{2}}\left|\mathcal{F}^{-1}\left(\mathcal{F} f \overline{\mathcal{F} \psi\left(A_{a} R_{\phi}^{-1} \cdot\right)}\right)(b)\right|^{2} \mathrm{~d} b\right) \mathrm{d} \phi \frac{\mathrm{d} a}{a} \\
& =\int_{\mathbb{R}^{2}}|\mathcal{F} f(\xi)|^{2}\left(\int_{[0,2 \pi) \times(0,1)}\left|\mathcal{F} \psi\left(A_{a} R_{\phi}^{-1} \xi\right)\right|^{2} \mathrm{~d} \phi \frac{\mathrm{d} a}{a}\right) \mathrm{d} \xi
\end{aligned}
$$

Thus, combining equations (37), (38) and (39) we obtain the reconstruction formula

$$
\|f\|^{2}=\int_{\mathbb{R}^{2}}\left|\left\langle f, T_{b} \Phi\right\rangle\right|^{2} \mathrm{~d} b+\int_{\mathbb{R}^{2} \rtimes([0,2 \pi) \times(0,1))}|\mathcal{G}(b, \phi, a)|^{2} \mathrm{~d} b \mathrm{~d} \phi \frac{\mathrm{d} a}{a^{5}} .
$$

It is worth observing that there always exists a function $\Phi$ satisfying (37) provided that the admissible vector $\psi$ has fast Fourier decay. Indeed, if we require $\mathcal{F} \psi$ to satisfy a decay estimate of the form

$$
|\mathcal{F} \psi(\xi)|=O\left(|\xi|^{-L}\right), \quad \text { for every } L>0,
$$

then, by (33) we have that

$$
\begin{aligned}
z(\xi) & :=1-\int_{[0,2 \pi) \times(0,1)}\left|\mathcal{F} \psi\left(A_{a} R_{\phi}^{-1} \xi\right)\right|^{2} \mathrm{~d} \phi \frac{\mathrm{d} a}{a} \\
& =\int_{[0,2 \pi) \times[1,+\infty)}\left|\mathcal{F} \psi\left(A_{a} R_{\phi}^{-1} \xi\right)\right|^{2} \mathrm{~d} \phi \frac{\mathrm{d} a}{a} \\
& \lesssim \int_{[0,2 \pi) \times[1,+\infty)} a^{-2 L}|\xi|^{-2 L} \frac{\mathrm{d} a}{a} \mathrm{~d} \phi \\
& \lesssim|\xi|^{-2 L} .
\end{aligned}
$$

Therefore, there exists a smooth function $\Phi$ such that $\mathcal{F} \Phi(\xi)=\sqrt{z(\xi)}$, so that (37) holds true.

Finally, let us show that the first term in the right hand side of (40) may be expressed in terms of $\mathcal{R}^{\text {pol }} f$ only, if we suppose that $\Phi \in \mathcal{A}^{\text {pol }}$. We readily derive

$$
\begin{aligned}
\left\langle f, T_{b} \Phi\right\rangle=\langle f, \pi(b, 0,1) \Phi\rangle & =\langle\mathcal{Q} f, \mathcal{Q} \pi(b, 0,1) \Phi\rangle \\
& =\langle\mathcal{Q} f, \hat{\pi}(b, 0,1) \mathcal{Q} \Phi\rangle \\
& =\left\langle\mathcal{I} \mathcal{R}^{\mathrm{pol}} f, \hat{\pi}(b, 0,1) \mathcal{I}^{\mathrm{pol}} \Phi\right\rangle \\
& =\left\langle\mathcal{R}^{\mathrm{pol}} f, \hat{\pi}(b, 0,1) \mathcal{I}^{2} \mathcal{R}^{\mathrm{pol}} \Phi\right\rangle,
\end{aligned}
$$

where we observe that $\mathcal{I} \mathcal{R}^{\text {pol }} \Phi$ is always in the domain of the operator $\mathcal{I}$ since

$$
\begin{aligned}
\int_{[0, \pi) \times \mathbb{R}}|\tau|\left|(I \otimes \mathcal{F}) \mathcal{I} \mathcal{R}^{\mathrm{pol}} \Phi(\theta, \tau)\right|^{2} \mathrm{~d} \theta \mathrm{d} \tau & =\int_{[0, \pi) \times \mathbb{R}}|\tau|^{2}\left|(I \otimes \mathcal{F}) \mathcal{R}^{\mathrm{pol}} \Phi(\theta, \tau)\right|^{2} \mathrm{~d} \theta \mathrm{d} \tau \\
& =\int_{[0, \pi) \times \mathbb{R}}|\tau|^{2}|\mathcal{F} \Phi(\tau w(\theta))|^{2} \mathrm{~d} \theta \mathrm{d} \tau \\
& =\int_{\mathbb{R}^{2}}|\xi||\mathcal{F} \Phi(\xi)|^{2} \mathrm{~d} \xi<+\infty
\end{aligned}
$$


since by definition $\Phi$ is a smooth function. Therefore, reconstruction formula (40) reads

$$
\|f\|^{2}=\int_{\mathbb{R}^{2}}\left|\left\langle\mathcal{R}^{\mathrm{pol}} f, \hat{\pi}(b, 0,1) \mathcal{I}^{2} \mathcal{R}^{\mathrm{pol}} \Phi\right\rangle\right|^{2} \mathrm{~d} b+\int_{\mathbb{R}^{2} \rtimes([0,2 \pi) \times(0,1))}|\mathcal{G}(b, \phi, a)|^{2} \mathrm{~d} b \mathrm{~d} \phi \frac{\mathrm{d} a}{a^{5}},
$$

where all the coefficients depend on $f$ only through its polar Radon transform.

It is worth observing that the domain of $\overline{\mathcal{R}^{\mathrm{pol}}}$ is related to the domain of $C$, which defines the admissible vectors of $\pi$. By Theorem廿 (ii), the operator $\left|\overline{\mathcal{R}^{\text {pol }}}\right|$ is a positive self-adjoint operator semi-invariant with weight $\chi(b, \phi, a)=a^{-1 / 2}$, which is a power of the modular function $\Delta(b, \phi, a)=a^{-2}$, i.e. $\chi(b, \phi, a)=\Delta(b, \phi, a)^{1 / 4}$. On the other hand, $C$ is a positive self-adjoint operator semi-invariant with weight $\Delta^{1 / 2}$ and is such that $\psi \in L^{2}\left(\mathbb{R}^{2}\right)$ is an admissible vector of the square-integrable representation $\pi$ if and only if $\psi \in \operatorname{dom} C$ and $\|C \psi\|=1$. Therefore, $\left|\overline{\mathcal{R}^{\text {pol }}}\right|$ and $C$ are both positive self-adjoint operators on $L^{2}\left(\mathbb{R}^{2}\right)$ semi-invariant with a power of the modular function of $\operatorname{SIM}(2)$ as weight. Finally, consider the subspace

$$
\mathcal{D}_{s}=\left\{f \in L^{2}\left(\mathbb{R}^{2}\right): \int_{\mathbb{R}^{2}}|\xi|^{2 s}|\mathcal{F} f(\xi)|^{2} \mathrm{~d} \xi<+\infty\right\}
$$

of $L^{2}\left(\mathbb{R}^{2}\right)$. It is not difficult to verify that the Fourier multiplier $A_{s}: \mathcal{D}_{s} \rightarrow L^{2}\left(\mathbb{R}^{2}\right)$ defined by

$$
\mathcal{F} A_{s} f(\xi)=|\xi|^{s} \mathcal{F} f(\xi)
$$

is a densely defined positive self-adjoint operator and is semi-invariant with weight $\chi_{s}(b, \phi, a)=\Delta(b, \phi, a)^{-s / 2}=a^{s}$. Thus, by Theorem [4, (i), the operators $\left|\overline{\mathcal{R}^{\text {pol }}}\right|$ and $C$ are given, up to a constant, by (42) with $s=-1 / 2$ and $s=-1$, respectively. The above argument explains why the domain of $\mathcal{R}^{\mathrm{pol}}$ and the domain of $C$, and thus the admissibility condition (33) of $\pi$, are strictly related. A similar result can be proved for the examples illustrated in Section 5 .

\section{$5 \quad$ Examples}

In this section, we illustrate two additional examples.

\subsection{The affine Radon transform and the shearlet transform}

\subsubsection{Groups and spaces}

The (parabolic) shearlet group $\mathbb{S}$ is the semidirect product of $\mathbb{R}^{2}$ with the closed subgroup $K=\left\{N_{s} A_{a} \in \mathrm{GL}(2, \mathbb{R}): s \in \mathbb{R}, a \in \mathbb{R}^{\times}\right\}$where

$$
N_{s}=\left[\begin{array}{cc}
1 & -s \\
0 & 1
\end{array}\right], \quad A_{a}=a\left[\begin{array}{cc}
1 & 0 \\
0 & |a|^{-1 / 2}
\end{array}\right] .
$$

We can identify the element $N_{s} A_{a}$ with the pair $(s, a)$ and we write $(b, s, a)$ for the elements in $\mathbb{S}$. With this identification the product law amounts to

$$
(b, s, a)\left(b^{\prime}, s^{\prime}, a^{\prime}\right)=\left(b+N_{s} A_{a} b^{\prime}, s+|a|^{1 / 2} s^{\prime}, a a^{\prime}\right)
$$

and the inverse of $(b, s, a)$ is given by

$$
(b, s, a)^{-1}=\left(-A_{a}^{-1} N_{s}^{-1} b,-|a|^{-1 / 2} s, a^{-1}\right) .
$$


A left Haar measure of $\mathbb{S}$ is

$$
\mathrm{d} \mu(b, s, a)=|a|^{-3} \mathrm{~d} b \mathrm{~d} s \mathrm{~d} a,
$$

with $\mathrm{d} b, \mathrm{~d} s$ and $\mathrm{d} a$ the Lebesgue measures on $\mathbb{R}^{2}, \mathbb{R}$ and $\mathbb{R}^{\times}$, respectively. The shearlet group acts transitively on $\Xi=\mathbb{R} \times \mathbb{R}$ by the action

$$
(b, s, a)^{-1} \cdot(v, t)=\left(|a|^{-1 / 2}(v-s), \frac{t-n(v) \cdot b}{a}\right)
$$

where $n(v)={ }^{t}(1, v)$. The isotropy at $\xi_{0}=(0,0)$ is

$$
H=\left\{\left(\left(0, b_{2}\right), 0, a\right): b_{2} \in \mathbb{R}, a \in \mathbb{R}^{\times}\right\},
$$

so that $\Xi=\mathbb{S} / H$. It is immediate to verify that the Lebesgue measure $\mathrm{d} \xi=\mathrm{d} v \mathrm{~d} t$ is a relatively invariant measure on $\Xi$ with positive character $\beta(b, s, a)=|a|^{3 / 2}$. Now, we consider the sections $s: \mathbb{R}^{2} \rightarrow \mathbb{S}$ and $\sigma: \mathbb{R} \times \mathbb{R} \rightarrow \mathbb{S}$ defined by

$$
s(x)=(x, 0,1), \quad \sigma(v, t)=((t, 0), v, 1) .
$$

Thus, we have that

$$
\begin{aligned}
& \hat{\xi}_{0}=H\left[x_{0}\right]=\left\{\left(0, b_{2}\right): b_{2} \in \mathbb{R}\right\} \simeq \mathbb{R}, \\
& \check{x}_{0}=K . \xi_{0}=\{(s, 0): s \in \mathbb{R}\} \simeq \mathbb{R} .
\end{aligned}
$$

It is easy to check that the Lebesgue measure $\mathrm{d} b_{2}$ on $\hat{\xi}_{0}$ is a relatively $H$-invariant measure with $\gamma\left(\left(0, b_{2}\right), 0, a\right)=|a|^{1 / 2}$. Further, we can compute

$$
\widehat{(v, t)}=\sigma(v, t)\left[\hat{\xi}_{0}\right]=\left\{x \in \mathbb{R}^{2}: x \cdot n(v)=t\right\},
$$

which is the set of all points laying on the line of equation $x \cdot n(v)=t$ and

$$
\check{x}=s(x) . \check{x}_{0}=\{(v, t) \in \mathbb{R} \times \mathbb{R}: t-n(v) \cdot x=0\},
$$

which parametrizes the set of all lines passing through the point $x$ except the horizontal one. Thus, the maps $x \mapsto \check{x}$ and $(v, t) \mapsto \widehat{(v, t)}$ are both injective. Therefore, $X=\mathbb{R}^{2}$ and $\Xi=\mathbb{R} \times \mathbb{R}$ are homogeneous spaces in duality.

\subsubsection{The representations}

The (parabolic) shearlet group $\mathbb{S}$ acts on $L^{2}\left(\mathbb{R}^{2}\right)$ via the shearlet representation, namely

$$
\pi(b, s, a) f(x)=|a|^{-3 / 4} f\left(A_{a}^{-1} N_{s}^{-1}(x-b)\right) .
$$

It is well known that the shearlet representation is irreducible [9].

Furthermore, since $\beta(b, s, a)=|a|^{3 / 2}$, the group $\mathbb{S}$ acts on $L^{2}(\mathbb{R} \times \mathbb{R}, \mathrm{d} v \mathrm{~d} t)$ by means of the quasi-regular representation $\hat{\pi}$ defined by

$$
\hat{\pi}(b, s, a) F(v, t)=|a|^{-\frac{3}{4}} F\left(|a|^{-1 / 2}(v-s), \frac{t-n(v) \cdot b}{a}\right) .
$$

By Mackey imprimitivity theorem [13], one can show that also $\hat{\pi}$ is irreducible. 


\subsubsection{The Radon transform}

By (11), the Radon transform between the homogeneous spaces in duality $\mathbb{R}^{2}$ and $\mathbb{R} \times \mathbb{R}$ is defined as

$$
\mathcal{R}^{\mathrm{aff}} f(v, t)=\int_{\mathbb{R}} f(t-v y, y) \mathrm{d} y,
$$

which is the so-called affine Radon transform [8, 17].

Following the same arguments as in Example 1, we define

$$
\mathcal{A}^{\text {aff }}=\left\{f \in L^{1}\left(\mathbb{R}^{2}\right) \cap L^{2}\left(\mathbb{R}^{2}\right): \int_{\mathbb{R}^{2}} \frac{|\mathcal{F} f(\xi)|^{2}}{\left|\xi_{1}\right|} \mathrm{d} \xi<+\infty\right\},
$$

where $\xi=\left(\xi_{1}, \xi_{2}\right) \in \mathbb{R}^{2}$, which is $\pi$-invariant and is such that $\mathcal{R}^{\text {aff }} f \in L^{2}(\mathbb{R} \times \mathbb{R})$ for all $f \in \mathcal{A}^{\text {aff }}$ (we refer to [5] for more details). Furthermore, as in Example 1, it is easy to show that $\mathcal{R}^{\text {aff }}$, regarded as operator from $\mathcal{A}^{\text {aff }}$ to $L^{2}(\mathbb{R} \times \mathbb{R})$, is closable.

\subsubsection{The Unitarization theorem}

Since $\beta(b, s, a)=|a|^{3 / 2}, \gamma(b, s, a)=|a|^{1 / 2}$ and $\left|\operatorname{det}\left(N_{s} A_{a}\right)\right|=|a|^{3 / 2}$ the affine Radon transform satisfies the intertwining property

$$
\mathcal{R}^{\mathrm{aff}} \pi(b, s, a)=\chi(b, s, a)^{-1} \hat{\pi}(b, s, a) \mathcal{R}^{\mathrm{aff}},
$$

where $\chi(b, \phi, a)^{-1}=|a|^{1 / 2}$.

By Lemma 3, the closure $\overline{\mathcal{R}^{\text {aff }}}$ of the affine Radon transform is a semi-invariant operator with weight $\chi(b, s, a)=|a|^{-1 / 2}$. Therefore, by Theorem 5 , there exists a positive selfadjoint operator $\mathcal{I}: \operatorname{dom}(\mathcal{I}) \subseteq L^{2}(\mathbb{R} \times \mathbb{R}) \rightarrow L^{2}(\mathbb{R} \times \mathbb{R})$ semi-invariant with weight $\zeta(g)=\chi(g)^{-1}=|a|^{1 / 2}$ such that $\mathcal{I R}^{\text {aff }}$ extends to a unitary operator $\mathcal{Q}$ from $L^{2}\left(\mathbb{R}^{2}\right)$ onto $L^{2}(\mathbb{R} \times \mathbb{R})$, which intertwines the quasi-regular (irreducible) representations $\pi$ and $\hat{\pi}$. Reasoning as in Example 1 it is possible to show that the operator $\mathcal{I}$ is defined by

$$
(I \otimes \mathcal{F}) \mathcal{I} f(\omega, \tau)=|\tau|^{\frac{1}{2}}(I \otimes \mathcal{F}) f(\omega, \tau), \quad f \in \mathcal{D},
$$

where

$$
\mathcal{D}=\left\{f \in L^{2}(\mathbb{R} \times \mathbb{R}): \int_{\mathbb{R} \times \mathbb{R}}|\tau||(I \otimes \mathcal{F}) f(\omega, \tau)|^{2} \mathrm{~d} \omega \mathrm{d} \tau<+\infty\right\}
$$

\subsubsection{The inversion formula}

It is known that the shearlet representation $\pi$ is square-integrable and its admissible vectors are the functions $\psi$ in $L^{2}\left(\mathbb{R}^{2}\right)$ satisfying

$$
\int_{\mathbb{R}^{2}} \frac{|\mathcal{F} \psi(\xi)|^{2}}{\left|\xi_{1}\right|^{2}} \mathrm{~d} \xi=1
$$

where $\xi=\left(\xi_{1}, \xi_{2}\right) \in \mathbb{R}^{2}$ [9. The shearlet transform is then $\mathcal{S}_{\psi} f(b, s, a)=\langle f, \pi(b, s, a) \psi\rangle$, and is a multiple of an isometry from $L^{2}\left(\mathbb{R}^{2}\right)$ into $L^{2}(\mathbb{S}, \mathrm{d} \mu)$ provided that $\psi$ satisfies the admissible condition (46). By Theorem [6] for any $f \in \mathcal{A}^{\text {aff }}$ we have the reconstruction formula

$$
f=\int_{\mathbb{R}^{2} \times \mathbb{R} \times \mathbb{R}^{\times}} \mathcal{S}_{\psi} f(b, s, a) \pi(b, s, a) \psi \frac{\mathrm{d} b \mathrm{~d} s \mathrm{~d} a}{|a|^{3}},
$$


where the coefficients $\mathcal{S}_{\psi} f(b, s, a)$ are given by

$$
\mathcal{S}_{\psi} f\left(b_{1}, b_{2}, s, a\right)=|a|^{-5 / 4} \int_{\mathbb{R} \times \mathbb{R}} \mathcal{R}^{\mathrm{aff}} f(v, t) \overline{\Psi\left(\frac{v-s}{|a|^{1 / 2}}, \frac{t-n(v) \cdot b}{a}\right)} \mathrm{d} v \mathrm{~d} t .
$$

If we choose $\Psi$ such that $\Psi(v, t)=\Psi_{2}(v) \Psi_{1}(t)$, then

$$
\mathcal{S}_{\psi} f\left(b_{1}, b_{2}, s, a\right)=|a|^{-3 / 4} \int_{\mathbb{R}} \mathcal{W}_{\Psi_{1}}\left(\mathcal{R}^{\mathrm{aff}} f(v, \cdot)\right)(n(v) \cdot b, a) \overline{\Psi_{2}\left(\frac{v-s}{|a|^{1 / 2}}\right)} \mathrm{d} v
$$

provided that $\Psi_{1}$ is a $1 \mathrm{D}$-wavelet.

This argument gives an alternative proof of Theorems 8 and 10 in [5, where it is also proved that formulas (48) can actually be extended to the whole $L^{1}\left(\mathbb{R}^{2}\right) \cap L^{2}\left(\mathbb{R}^{2}\right)$ and it is not difficult to verify that this extension works in the same way for Example 1. Formula (47) is a continuous version of the reconstruction formula presented in 8 , Theorem 3.3].

\subsection{The spherical means Radon transform}

\subsubsection{Groups and spaces}

Take the same group $G=S I M(2)$ as in Example 1] namely $G=\mathbb{R}^{2} \rtimes K$, with $K=\left\{R_{\phi} A_{a} \in \mathrm{GL}(2, \mathbb{R}): \phi \in[0,2 \pi), a \in \mathbb{R}^{+}\right\}$. We consider the space $\Xi=\mathbb{R}^{2} \times \mathbb{R}^{+}$, which we think of as parametrizing centers and radii of circles in $\mathbb{R}^{2}$, with the action

$$
(b, \phi, a) \cdot(c, r)=\left(b+a R_{\phi} c, a r\right) .
$$

An immediate calculation shows that the isotropy at $\xi_{0}=((1,0), 1)$ is

$$
H=\{((1-\cos \phi,-\sin \phi), \phi, 1): \phi \in[0,2 \pi)\} .
$$

By direct computation, recalling that $x_{0}=0$,

$$
\hat{\xi}_{0}=H\left[x_{0}\right]=\{(1-\cos \phi,-\sin \phi): \phi \in[0,2 \pi)\}
$$

is the circle with center $(1,0)$ and radius 1 and

$$
\check{x}_{0}=K . \xi_{0}=\left\{((a \cos \phi, a \sin \phi), a): \phi \in[0,2 \pi), a \in \mathbb{R}^{+}\right\}
$$

is the family of circles passing through the origin. The measure $\mathrm{d} m_{0}=\mathrm{d} \phi$ is $H$ invariant on $\hat{\xi}_{0}$, since the action of $H$ on $\hat{\xi}_{0}$ is given by a simple rotation of a fixed angle. This gives $\gamma(h) \equiv 1$.

We define the section $\sigma: \Xi \rightarrow \operatorname{SIM}(2)$ by $\sigma(c, r)=(c-(r, 0), 0, r)$. Thus, for $\xi=(c, r) \in \Xi$ we have

$$
\hat{\xi}=\sigma(c, r)\left[\hat{\xi}_{0}\right]=\{c-r w(\phi): \phi \in[0,2 \pi)\},
$$

namely, the circle with center $c$ and radius $r$ and, for $x \in \mathbb{R}^{2}$ we have

$$
\check{x}=s(x) . \check{x}_{0}=\left\{(x+(a \cos \phi, a \sin \phi), a): \phi \in[0,2 \pi), a \in \mathbb{R}^{+}\right\},
$$

that is, the family of circles passing through the point $x$. It is easy to see that the maps $x \mapsto \check{x}$ and $\xi \mapsto \hat{\xi}$ are both injective. Thus, $X=\mathbb{R}^{2}$ and $\Xi=\mathbb{R}^{2} \times \mathbb{R}^{+}$are homogeneous spaces in duality. 
We now fix a relatively invariant measure on $\Xi$ : as we will show, this requires some care. Given $\alpha \in \mathbb{R}$, we have

$$
\int_{\mathbb{R}^{2} \times \mathbb{R}^{+}} f\left((b, \phi, a)^{-1} \cdot(c, r)\right) \mathrm{d} c \frac{\mathrm{d} r}{r^{\alpha}}=a^{3-\alpha} \int_{\mathbb{R}^{2} \times \mathbb{R}^{+}} f(c, r) \mathrm{d} c \frac{\mathrm{d} r}{r^{\alpha}},
$$

so that the measure $\mathrm{d} \xi=\mathrm{d} c \frac{\mathrm{d} r}{r^{\alpha}}$ is a relatively invariant measure on $\Xi$ with character $\beta(b, \phi, a)=a^{3-\alpha}$.

\subsubsection{The representations}

Since the group $G$ is the same as in Example 1, the representation $\pi$ is given by (8), whereas we have to compute the quasi-regular representation $\hat{\pi}$ acting on $L^{2}(\Xi, \mathrm{d} \xi)$. Since $\beta(b, \phi, a)=a^{3-\alpha}$, by (6) and (49) we have

$$
\begin{aligned}
\hat{\pi}(b, \phi, a) F(c, r) & =a^{\frac{\alpha-3}{2}} F\left(\left(-A_{a}^{-1} R_{\phi}^{-1} b,-\phi \bmod 2 \pi, a^{-1}\right) \cdot(c, r)\right) \\
& =a^{\frac{\alpha-3}{2}} F\left(a^{-1} R_{-\phi}(c-b), a^{-1} r\right),
\end{aligned}
$$

which is irreducible by Mackey imprimitivity theorem [13].

\subsubsection{The Radon transform}

By (50) and (11), the Radon trasform in this case is given by

$$
\mathcal{R}^{\mathrm{cir}} f(c, r)=\int_{0}^{2 \pi} f(c-r w(\phi)) \mathrm{d} \phi,
$$

namely, the integral of $f$ over the circle of center $c$ and radius $r$. This is the so-called spherical means Radon transform [25]. It is worth observing that more interesting problems arise when the available centers and radii are restricted to some hypersurface: this does not easily fit into our assumptions, and it is left for future investigation.

Let us now determine a suitable $\pi$-invariant subspace $\mathcal{A}^{\text {cir }}$ of $L^{2}\left(\mathbb{R}^{2}\right)$ as in (A7) In order to do that, it is useful to derive a Fourier slice theorem for $\mathcal{R}^{\text {cir }}$. For $f \in$ $L^{1}\left(\mathbb{R}^{2}\right) \cap L^{2}\left(\mathbb{R}^{2}\right)$, by Fubini's theorem and using [11, Eq. 10.9.1], we have

$$
\begin{aligned}
(\mathcal{F} \otimes I) \mathcal{R}^{\mathrm{cir}} f(\tau, r) & =\int_{0}^{2 \pi} \int_{\mathbb{R}^{2}} f(c-r w(\phi)) e^{-2 \pi i c \cdot \tau} \mathrm{d} c \mathrm{~d} \phi \\
& =\int_{0}^{2 \pi} e^{-2 \pi i r w(\phi) \cdot \tau} \mathrm{d} \phi \int_{\mathbb{R}^{2}} f(c) e^{-2 \pi i c \cdot \tau} \mathrm{d} c \\
& =2 \pi J_{0}(2 \pi|\tau| r) \mathcal{F} f(\tau),
\end{aligned}
$$

where $J_{0}$ is the Bessel function of the first kind. As a consequence, by Plancherel theorem, recalling that $\mathrm{d} \xi=\mathrm{d} c \frac{\mathrm{d} r}{r^{\alpha}}$ we obtain

$$
\left\|\mathcal{R}^{\mathrm{cir}} f\right\|_{L^{2}(\Xi, \mathrm{d} \xi)}^{2}=\left\|(\mathcal{F} \otimes I) \mathcal{R}^{\mathrm{cir}} f\right\|_{L^{2}\left(\Xi, \mathrm{d} \tau \frac{\mathrm{d} r}{r \alpha}\right)}^{2}=c_{\alpha} \int_{\mathbb{R}^{2}}|\mathcal{F} f(\tau)|^{2}|\tau|^{\alpha-1} \mathrm{~d} \tau,
$$

where

$$
c_{\alpha}=(2 \pi)^{\alpha+1} \int_{\mathbb{R}^{+}} \frac{\left|J_{0}(r)\right|^{2}}{r^{\alpha}} \mathrm{d} r .
$$


Observe that $c_{\alpha}$ is finite if and only if $\alpha \in(0,1)$, so that from now on we assume that $\alpha \in(0,1)$ and we set

$$
\mathcal{A}_{\alpha}^{\text {cir }}=\left\{f \in L^{1}\left(\mathbb{R}^{2}\right) \cap L^{2}\left(\mathbb{R}^{2}\right): \int_{\mathbb{R}^{2}}|\mathcal{F} f(\tau)|^{2}|\tau|^{\alpha-1} \mathrm{~d} \tau<+\infty\right\},
$$

which is $\pi$-invariant and is such that $\mathcal{R}^{\text {cir }} f \in L^{2}(\Xi, \mathrm{d} \xi)$ for all $f \in \mathcal{A}_{\alpha}^{\text {cir }}$. Furthermore, as in Example 1 it is easy to show that $\mathcal{R}^{\text {cir }}$, regarded as operator from $\mathcal{A}_{\alpha}^{\text {cir }}$ to $L^{2}(\Xi, \mathrm{d} \xi)$, is closable. We stress that, if $\alpha \notin(0,1)$, the set

$$
\left\{f \in L^{2}(X, \mathrm{~d} x): \mathcal{R}^{\text {cir }} f \in L^{2}(\Xi, \mathrm{d} \xi)\right\}=\{0\},
$$

i.e. it is trivial. This motivates the role of Assumption (A7) in our construction.

\subsubsection{The Unitarization theorem}

By (14) and (15) we have that

$$
\mathcal{R}^{\mathrm{cir}} \pi(b, \phi, a)=a^{\frac{1-\alpha}{2}} \hat{\pi}(b, \phi, a) \mathcal{R}^{\mathrm{cir}},
$$

since $\beta(b, \phi, a)=a^{3-\alpha}, \gamma(b, \phi, a)=1$ and $\operatorname{det}\left(R_{\phi} A_{a}\right)=a^{2}$, and so $\chi(b, \phi, a)=a^{\frac{\alpha-1}{2}}$.

By Theorem [5, there exists a positive self-adjoint operator $\mathcal{I}$, semi-invariant with weight $a^{\frac{1-\alpha}{2}}$, such that $\mathcal{I} \mathcal{R}^{\text {cir }}$ extends to a unitary operator $\mathcal{Q}: L^{2}\left(\mathbb{R}^{2}\right) \rightarrow L^{2}(\Xi, \mathrm{d} \xi)$. Moreover, $\mathcal{Q}$ intertwines $\pi$ and $\hat{\pi}$, namely

$$
\hat{\pi}(b, \phi, a) \mathcal{Q} \pi(b, \phi, a)^{-1}=\mathcal{Q}, \quad(b, \phi, a) \in \operatorname{SIM}(2) .
$$

As in the other examples, by using Theorem 4 part (i), it is possible to show that there exists a constant $k_{\alpha} \in \mathbb{R}^{+}$such that $\mathcal{I}=k_{\alpha} \mathcal{J}$ with

$$
(\mathcal{F} \otimes I) \mathcal{J} f(\tau, r)=|\tau|^{\frac{1-\alpha}{2}}(\mathcal{F} \otimes I) f(\tau, r), \quad f \in \mathcal{D},
$$

where

$$
\mathcal{D}=\left\{f \in L^{2}(\Xi, \mathrm{d} \xi): \int_{\mathbb{R}^{2} \times \mathbb{R}^{+}}|\tau|^{1-\alpha}|(\mathcal{F} \otimes I) f(\tau, r)|^{2} \mathrm{~d} \tau \frac{\mathrm{d} r}{r^{\alpha}}<+\infty\right\} .
$$

Using the same argument as in Example 1 it is possible to determine the constant $k_{\alpha}$. Take a function $f \in \mathcal{A}_{\alpha}^{\text {cir }} \backslash\{0\}$. By Plancherel theorem and the Fourier slice theorem obtained for $\mathcal{R}^{\text {cir }}$ we have that

$$
\begin{aligned}
\|f\|^{2}=\left\|\mathcal{I} \mathcal{R}^{\mathrm{cir}} f\right\|_{L^{2}\left(\mathbb{R}^{2} \times \mathbb{R}^{+}\right)}^{2} & =k_{\alpha}^{2}\left\|(\mathcal{F} \otimes I) \mathcal{J} \mathcal{R}^{\mathrm{cir}} f\right\|_{L^{2}\left(\mathbb{R}^{2} \times \mathbb{R}^{+}\right)}^{2} \\
& =k_{\alpha}^{2} \int_{\mathbb{R}^{2} \times \mathbb{R}^{+}}|\tau|^{1-\alpha}\left|(\mathcal{F} \otimes I) \mathcal{R}^{\mathrm{cir}} f(\tau, r)\right|^{2} \mathrm{~d} \tau \frac{\mathrm{d} r}{r^{\alpha}} \\
& =k_{\alpha}^{2} c_{\alpha}\|f\|^{2},
\end{aligned}
$$

where $c_{\alpha}$ is given by (52). Thus, we obtain that $k_{\alpha}=c_{\alpha}^{-1 / 2}$.

\subsubsection{The inversion formula}

Applying Theorem 6 to $\mathcal{R}^{\text {cir }}$ we obtain the inversion formula for $f \in \mathcal{A}_{\alpha}^{\text {cir }}$

$$
f=\int_{S I M(2)} a^{\frac{\alpha-9}{2}}\left\langle\mathcal{R}^{\mathrm{cir}} f, \hat{\pi}(b, \phi, a) \Psi\right\rangle_{L^{2}(\Xi, \mathrm{d} \xi)} \psi\left(R_{-\phi} \frac{x-b}{a}\right) \mathrm{d} b \mathrm{~d} \phi \mathrm{d} a,
$$

where we used that $\chi(b, \phi, a)=a^{\frac{\alpha-1}{2}}$, the expression for the Haar measure of $\operatorname{SIM}(2)$ given in (7) and the expression for the representation $\pi$ given in (8). 


\section{References}

[1] G. S. Alberti, L. Balletti, F. De Mari, and E. De Vito. Reproducing subgroups of $S p(2, \mathbb{R})$. Part I: Algebraic classification. J. Fourier Anal. Appl., 19(4):651-682, 2013.

[2] G. S. Alberti, S. Dahlke, F. De Mari, E. De Vito, and H. Führ. Recent progress in shearlet theory: systematic construction of shearlet dilation groups, characterization of wavefront sets, and new embeddings. In Frames and other bases in abstract and function spaces, Appl. Numer. Harmon. Anal., pages 127-160. Birkhäuser/Springer, Cham, 2017.

[3] G. S. Alberti, F. De Mari, E. De Vito, and L. Mantovani. Reproducing subgroups of $\operatorname{Sp}(2, \mathbb{R})$. Part II: admissible vectors. Monatsh. Math., 173(3):261-307, 2014.

[4] J.-P. Antoine and R. Murenzi. Two-dimensional directional wavelets and the scale-angle representation. Signal processing, 52(3):259-281, 1996.

[5] F. Bartolucci, F. De Mari, E. De Vito, and F. Odone. The Radon transform intertwines wavelets and shearlets. Applied and Computational Harmonic Analysis, 2018. (available on line https://doi.org/10.1016/j.acha.2017.12.005).

[6] A. Ben-Israel and T. N. E. Greville. Generalized inverses, volume 15 of CMS Books in Mathematics/Ouvrages de Mathématiques de la SMC. Springer-Verlag, New York, 2nd edition, 2003. Theory and applications.

[7] C. Berenstein and D. Walnut. Local inversion of the Radon transform in even dimensions using wavelets. In 75 years of Radon transform (Vienna, 1992), Conf. Proc. Lecture Notes Math. Phys., IV, pages 45-69. Int. Press, Cambridge, MA, 1994.

[8] F. Colonna, G. Easley, K. Guo, and D. Labate. Radon transform inversion using the shearlet representation. Applied and Computational Harmonic Analysis, 29(2):232-250, 2010.

[9] S. Dahlke, G. Kutyniok, P. Maass, C. Sagiv, H. Stark, and G. Teschke. The uncertainty principle associated with the continuous shearlet transform. International Journal of Wavelets, Multiresolution and Information Processing, 6(02):157-181, 2008 .

[10] S. Dahlke, G. Steidl, and G. Teschke. The continuous shearlet transform in arbitrary space dimensions. Journal of Fourier Analysis and Applications, 16(3):340$364,2010$.

[11] NIST Digital Library of Mathematical Functions. http://dlmf.nist.gov/, Release 1.0.20 of 2018-09-15. F. W. J. Olver, A. B. Olde Daalhuis, D. W. Lozier, B. I. Schneider, R. F. Boisvert, C. W. Clark, B. R. Miller and B. V. Saunders, eds.

[12] M. Duflo and C. C. Moore. On the regular representation of a nonunimodular locally compact group. J. Functional Analysis, 21(2):209-243, 1976.

[13] G. B. Folland. A course in abstract harmonic analysis. Textbooks in Mathematics. CRC Press, Boca Raton, FL, second edition, 2016.

[14] H. Führ. Continuous wavelet transforms with abelian dilation groups. J. Math. Phys., 39(8):3974-3986, 1998.

[15] H. Führ. Abstract harmonic analysis of continuous wavelet transforms. Springer, 2005. 
[16] H. Führ and R. R. Tousi. Simplified vanishing moment criteria for wavelets over general dilation groups, with applications to abelian and shearlet dilation groups. Applied and Computational Harmonic Analysis, 2016.

[17] P. Grohs. Continuous shearlet frames and resolution of the wavefront set. Monatshefte für Mathematik, 164(4):393-426, 2011.

[18] S. Helgason. Radon-Fourier transforms on symmetric spaces and related group representations. Bull. Amer. Math. Soc., 71:757-763, 1965.

[19] S. Helgason. The Radon transform on Euclidean spaces, compact two-point homogeneous spaces and Grassmann manifolds. Acta Math., 113:153-180, 1965.

[20] S. Helgason. The Radon transform, volume 5 of Progress in Mathematics. Birkhäuser Boston, Inc., Boston, MA, 2nd edition, 1999.

[21] S. Helgason. Groups and geometric analysis, volume 83 of Mathematical Surveys and Monographs. American Mathematical Society, Providence, RI, 2000. Integral geometry, invariant differential operators, and spherical functions, Corrected reprint of the 1984 original.

[22] S. Helgason. Geometric analysis on symmetric spaces, volume 39 of Mathematical Surveys and Monographs. American Mathematical Society, Providence, RI, 2nd edition, 2008.

[23] S. Helgason. Integral geometry and Radon transforms. Springer, New York, 2011.

[24] M. Holschneider. Inverse Radon transforms through inverse wavelet transforms. Inverse Problems, 7(6):853-861, 1991.

[25] P. Kuchment and L. Kunyansky. Mathematics of thermoacoustic tomography. European J. Appl. Math., 19(2):191-224, 2008.

[26] D. Labate, W.-Q. Lim, G. Kutyniok, and G. Weiss. Sparse multidimensional representation using shearlets. In Optics 83 Photonics 2005, pages 59140U-59140U. International Society for Optics and Photonics, 2005.

[27] W. R. Madych. Tomography, approximate reconstruction, and continuous wavelet transforms. Appl. Comput. Harmon. Anal., 7(1):54-100, 1999.

[28] T. Olson and J. DeStefano. Wavelet localization of the radon transform. IEEE Transactions on Signal Processing, 42(8):2055-2067, Aug 1994.

[29] A. G. Ramm and A. I. Katsevich. The Radon transform and local tomography. CRC Press, Boca Raton, FL, 1996.

[30] V. S. Varadarajan. Geometry of quantum theory. Springer-Verlag, New York, 2nd edition, 1985.

[31] D. Walnut. Local inversion of the radon transform in the plane using wavelets. Proc. SPIE, 2034:84-90, 1993. 